\title{
Thoracic and cardiovascular surgeries in Japan during 2018
}

\author{
Annual report by the Japanese Association for Thoracic Surgery
}

\author{
Committee for Scientific Affairs, The Japanese Association for Thoracic Surgery ${ }^{1} \cdot$ Hideyuki Shimizu $^{2}$. \\ Morihito Okada ${ }^{3} \cdot$ Yasushi Toh $^{4} \cdot$ Yuichiro Doki $^{5} \cdot$ Shunsuke Endo $^{6} \cdot$ Hirotsugu Fukuda $^{7}$. \\ Yasutaka Hirata $^{8} \cdot$ Hisashi Iwata $^{9} \cdot$ Junjiro Kobayashi $^{10} \cdot$ Hiraku Kumamaru $^{11} \cdot$ Hiroaki Miyata $^{12}$. \\ Noboru Motomura $^{13}$. Shoji Natsugoe ${ }^{14}$. Soji Ozawa ${ }^{15} \cdot$ Yoshikatsu Saiki $^{16} \cdot$ Aya Saito $^{17}$. \\ Hisashi Saji $^{18} \cdot$ Yukio Sato $^{19} \cdot$ Tsuyoshi Taketani $^{20} \cdot$ Kazuo Tanemoto $^{21}$ - Akira Tangoku ${ }^{22}$. \\ Wataru Tatsuishi ${ }^{23} \cdot$ Hiroyuki Tsukihara $^{24}$ - Masayuki Watanabe ${ }^{25}$. Hiroyuki Yamamoto ${ }^{26}$. \\ Kenji Minatoya $^{27} \cdot$ Kohei Yokoi $^{28} \cdot$ Yutaka Okita $^{29} \cdot$ Masanori Tsuchida $^{30} \cdot$ Yoshiki Sawa $^{31}$
}

The Japanese Association for Thoracic Surgery has conducted annual surveys of thoracic surgery throughout Japan since 1986 to determine statistics pertaining to the number of procedures performed according to surgical categories. We herein summarize the results of the association's annual survey of thoracic surgeries performed in 2018.

Adhering to the norm thus far, thoracic surgery had been classified into three categories, cardiovascular, general thoracic, and esophageal surgeries, with patient data for each group being examined and analyzed. We honor and value all members' continued professional support and contributions.

Incidence of hospital mortality was included in the survey to determine nationwide status, which has contributed to Japanese surgeons' understanding of the present status of thoracic surgery in Japan while helping to effect improvements in surgical outcomes by enabling comparisons between their work and that of others. This approach has enabled the association to gain a better understanding of present problems and future prospects, which is reflected in its activities and member education.

Morihito Okada and Akira Tangoku have contributed equally to this work.

Annual report by the Japanese Association for Thoracic Surgery: Committee for Scientific Affairs.

Hideyuki Shimizu

survey-adm@umin.net

Extended author information available on the last page of the article
Thirty-day mortality (otherwise known as operative mortality) is defined as death within 30 days of surgery, regardless of the patient's geographic location, including post-discharge from the hospital. Hospital mortality is defined as death within any time interval following surgery among patients yet to be discharged from the hospital.

While hospital-to-hospital transfer during esophageal surgery is not considered a form of discharge, transfer to a nursing home or a rehabilitation unit is considered hospital discharge, unless the patient subsequently dies of complications from surgery. In contrast, hospital-to-hospital transfer 30 days following cardiovascular and general thoracic surgeries is considered discharge given that data related to the National Clinical Database (NCD) were employed in these categories.

\section{Survey abstract}

All data pertaining to cardiovascular and thoracic surgeries were obtained from the NCD, whereas data regarding esophageal surgery were collected from a survey questionnaire derived from the Japanese Association for Thoracic Surgery documentation. This is because NCD information regarding esophageal surgery does not include non-surgical cases (i.e., patients with adjuvant chemotherapy or radiation only).

Given the changes in data collection related to cardiovascular surgery [initially self-reported using questionnaire sheets in each participating institution up to 2014, followed by downloading of an automatic package from the Japanese Cardiovascular Surgery Database (JCVSD), a 
Table 1 Number of institutions involved in the survey

\begin{tabular}{llll}
\hline & \multicolumn{2}{l}{ Questionnaires } & \\
\cline { 2 - 4 } & Sent out & Responded & Response rate \\
\hline (A) Cardiovascular surgery & & & \\
(B) General Thoracic Surgery & 749 & 676 & $90.3 \%$ \\
(C) Esophageal surgery & & 552 & \\
\hline
\end{tabular}

Table 2 Categories subclassified according to the number of operations performed

\begin{tabular}{ll}
\hline Number of operations performed & $\begin{array}{l}\text { Category } \\
\text { General thoracic surgery }\end{array}$ \\
\hline 0 & 5 \\
$1-24$ & 38 \\
$25-49$ & 94 \\
$50-99$ & 193 \\
$100-149$ & 121 \\
$150-199$ & 107 \\
$\geq 200$ & 118 \\
Total & 676 \\
\hline
\end{tabular}

cardiovascular subsection of the $\mathrm{NCD}$ ], response rates were unavailable and were therefore not indicated in the cardiovascular surgery category (Table 1). Additionally, the number of institutions (based on surgery count) was not calculated in the cardiovascular surgery category (Table 2).

\section{Final report: 2018}

\section{(A) Cardiovascular surgery}

We are extremely pleased with the cooperation of our colleagues (members) in completing the cardiovascular surgery survey, which has undoubtedly improved the quality of this annual report. We are truly grateful for the significant efforts made by all participants within each participating institution in completing the JCVSD/NCD.

Figure 1 illustrates the development of cardiovascular surgery in Japan over the past 32 years. Aneurysm surgery includes only surgeries for thoracic and thoracoabdominal aortic aneurysms. Extra-anatomic bypass surgery for thoracic aneurysm and pacemaker implantation have been excluded from the survey since 2015. Assist device implantations were not included in the total number of surgical procedures but were nonetheless included in the survey.

A total of 69,063 cardiovascular surgeries, including 51 heart transplants, had been performed in 2018, a decrease of $0.7 \%$ compared to that in $2017(\mathrm{n}=70,078)$.

Compared to data for 2017 [1] and 2008 [2], data for 2018 showed $1.2 \%$ (9253 vs. 9368) and 3.6\% fewer surgeries for congenital heart disease, $0.5 \%(23,205$ vs. 23,312$)$ fewer and $38.6 \%$ more surgeries for valvular heart disease, $12.7 \%(12,135$ vs. 13,898$)$ and $36.9 \%$ fewer surgeries for ischemic heart procedures, and $4.2 \%$ (21,624 vs. 20,746) and $96.6 \%$ more surgeries for thoracic aortic aneurysm, respectively. Data for individual categories are summarized in Tables 3, 4, 5, 6, 7, 8 .

\section{Cardiovascular Surgery}

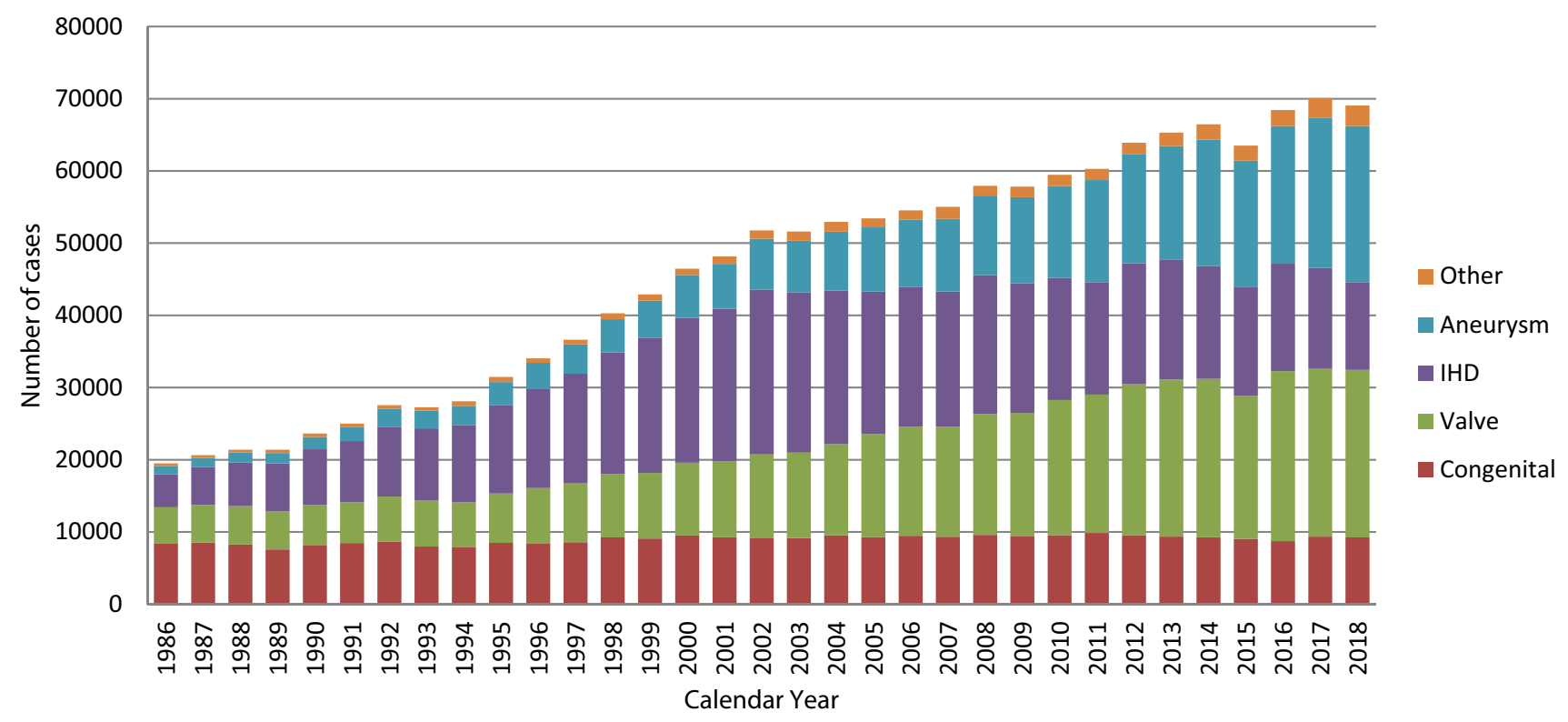

Fig. 1 Cardiovascular surgery. IHD ischemic heart disease 


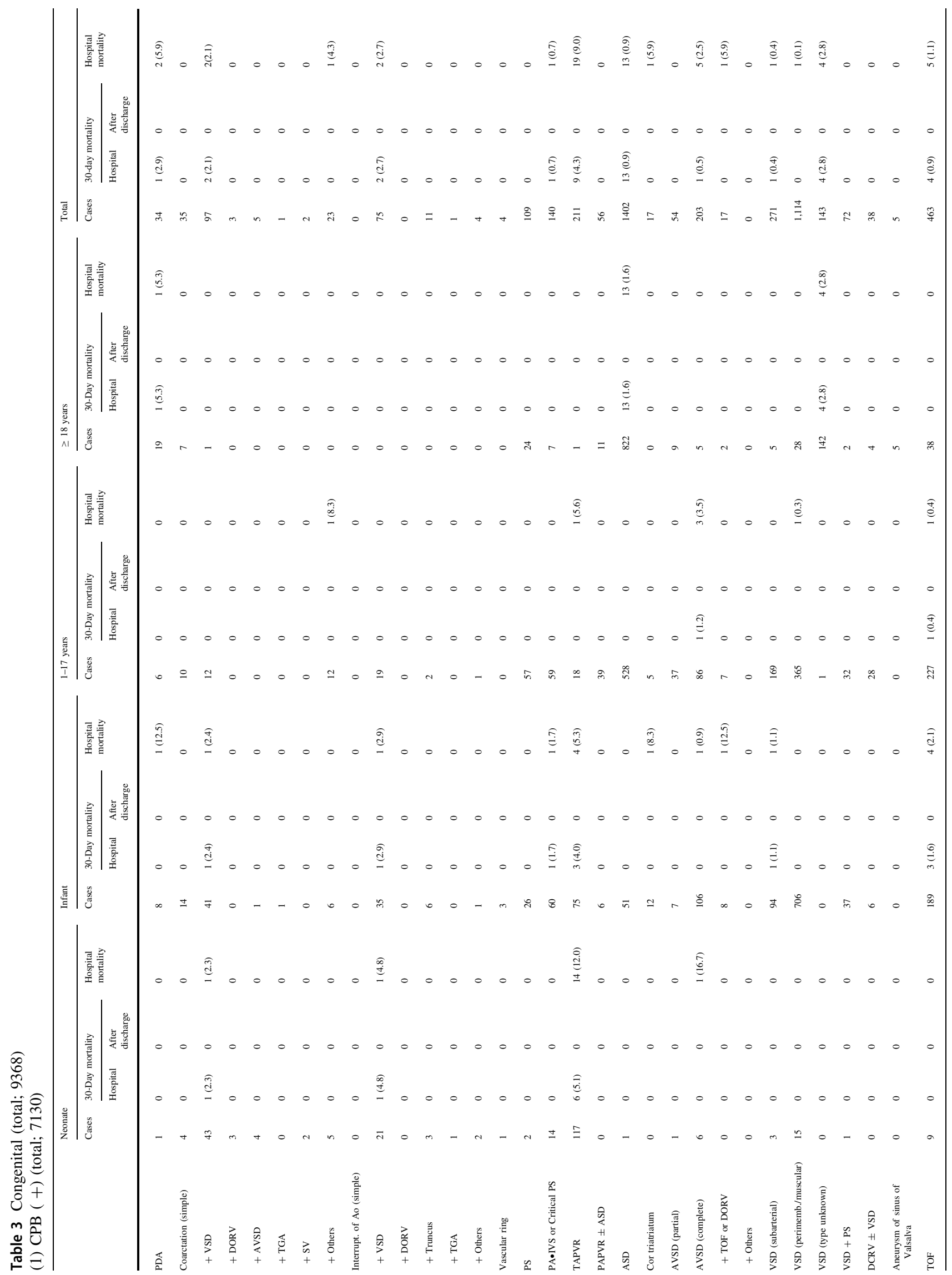


182

General Thoracic and Cardiovascular Surgery (2021) 69:179-212

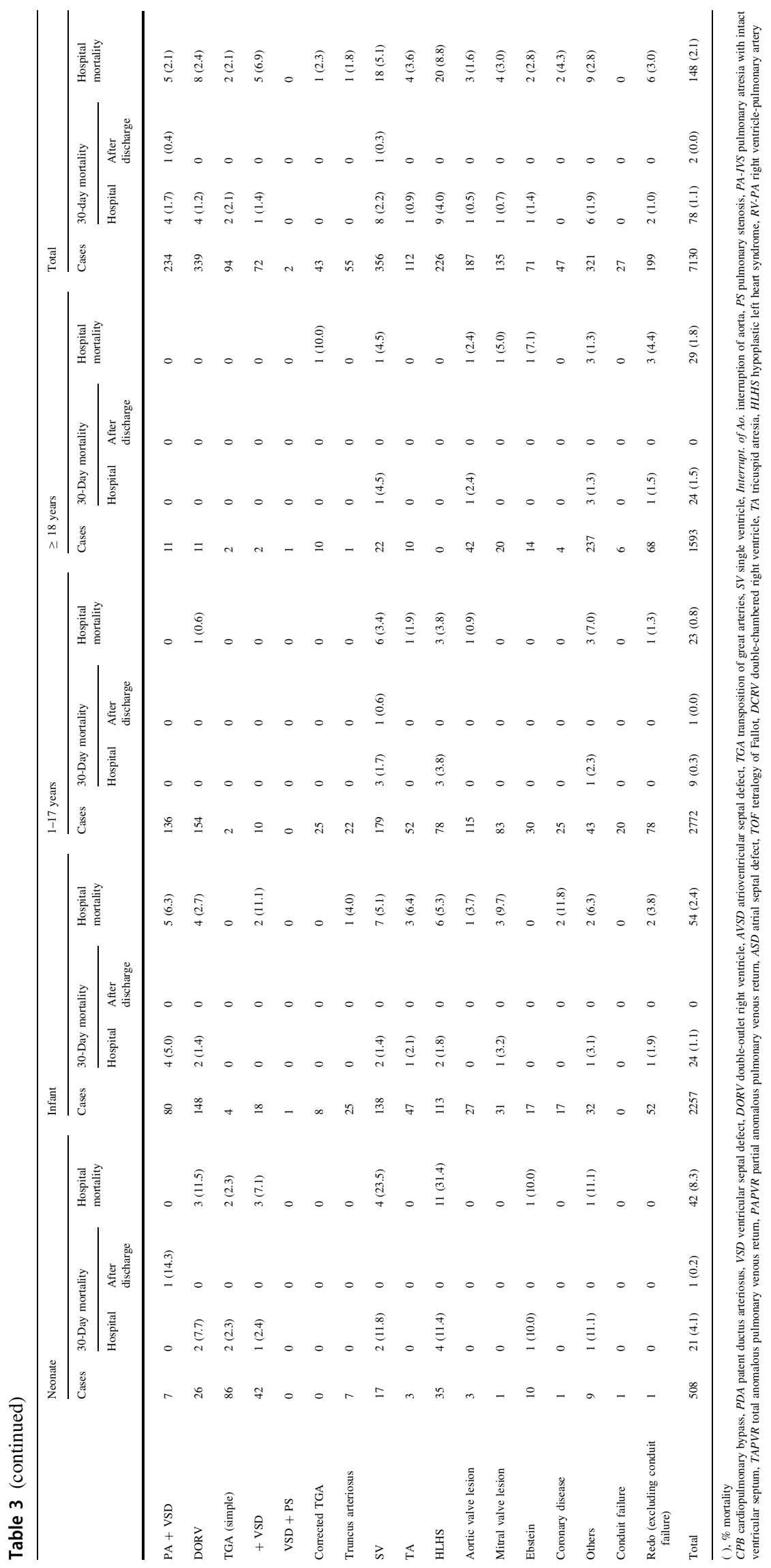

Springer 


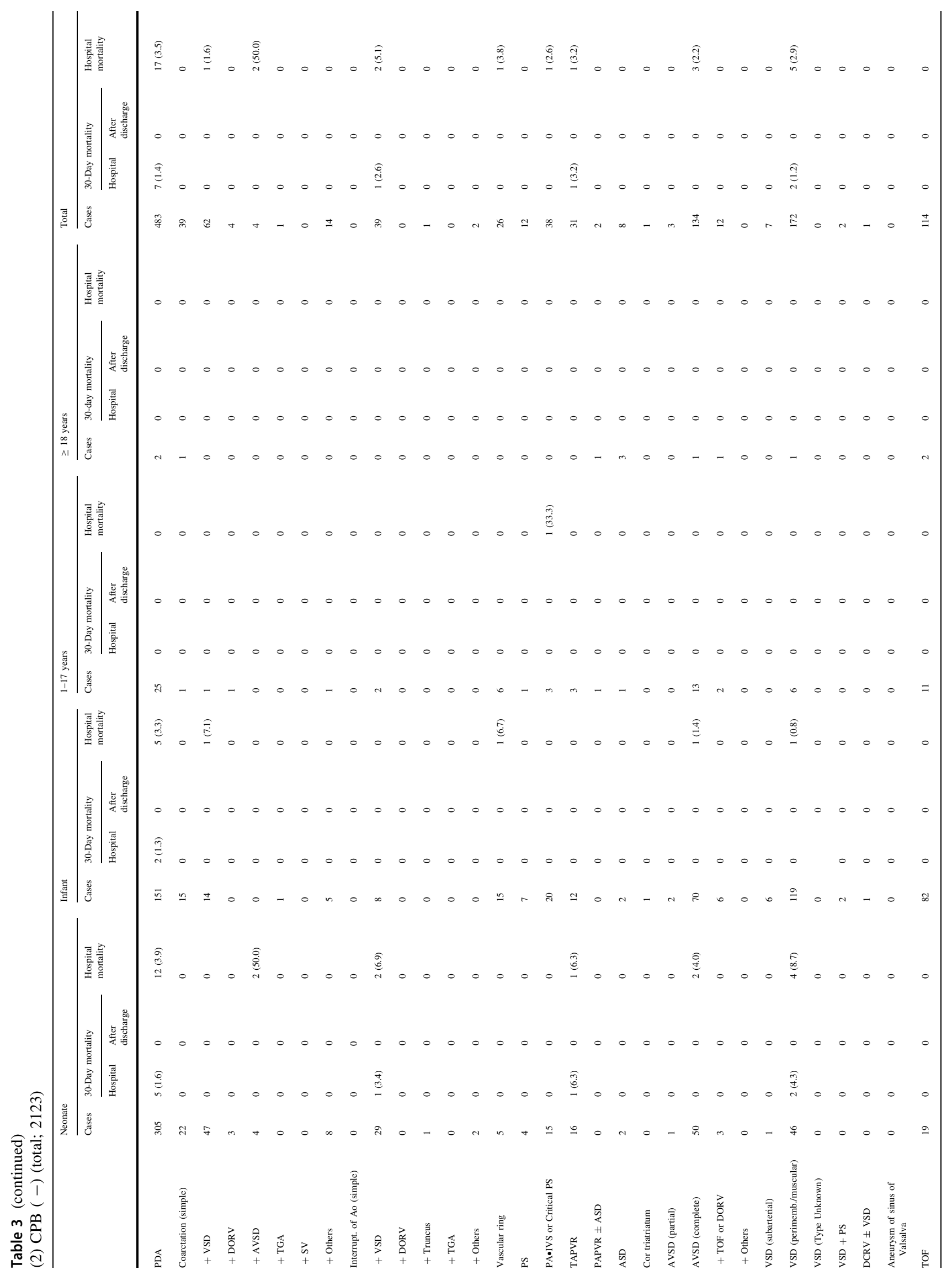




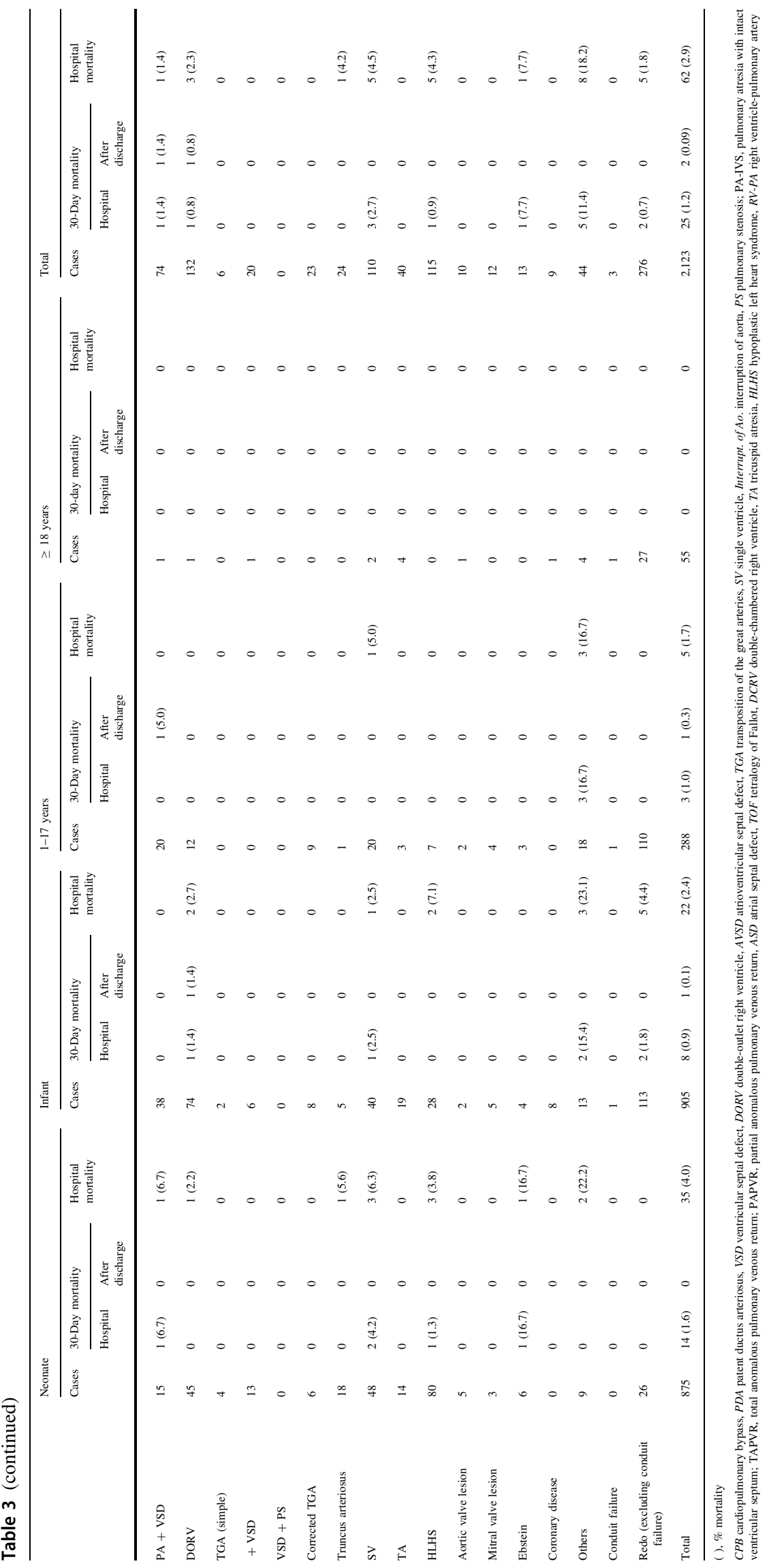




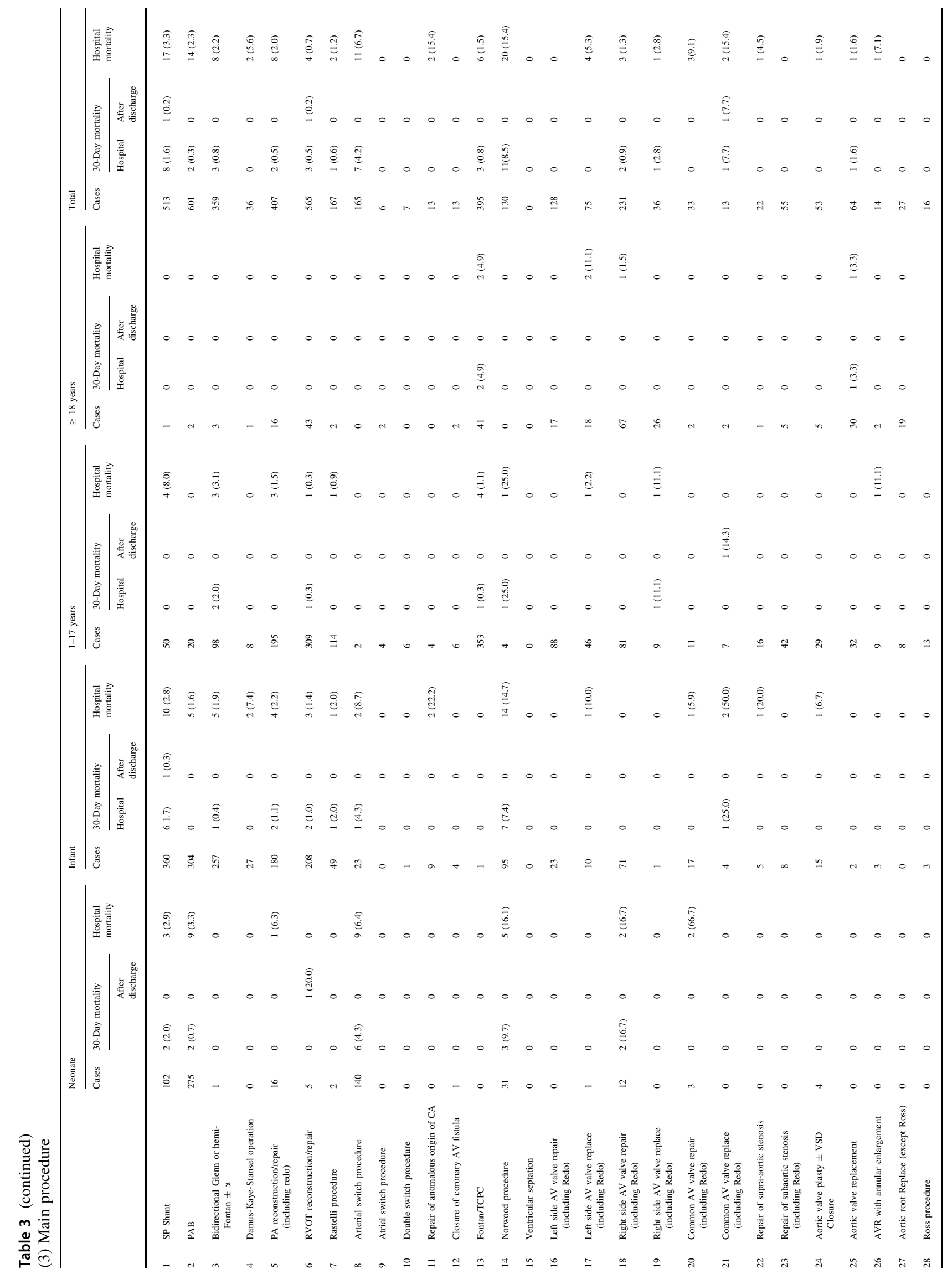




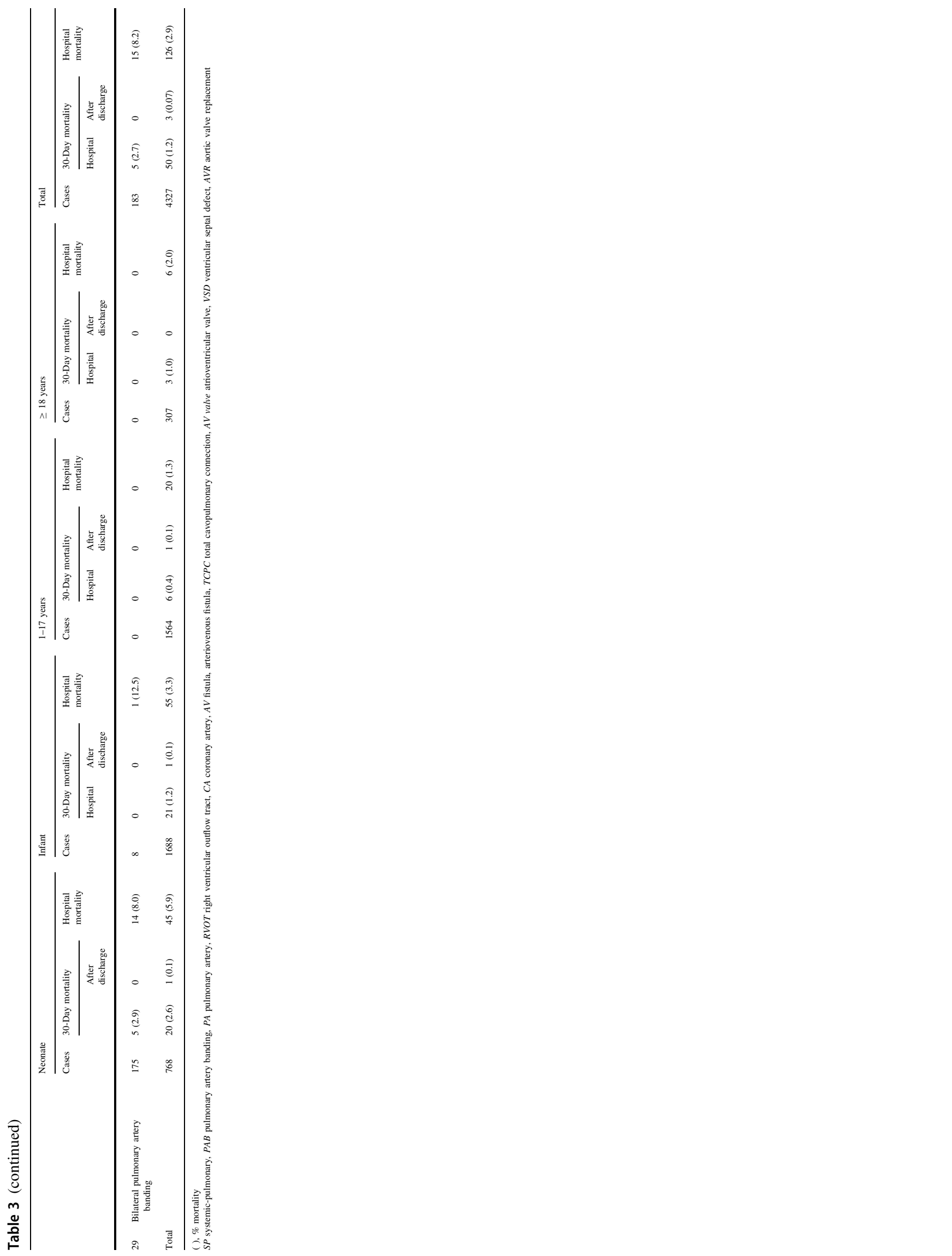




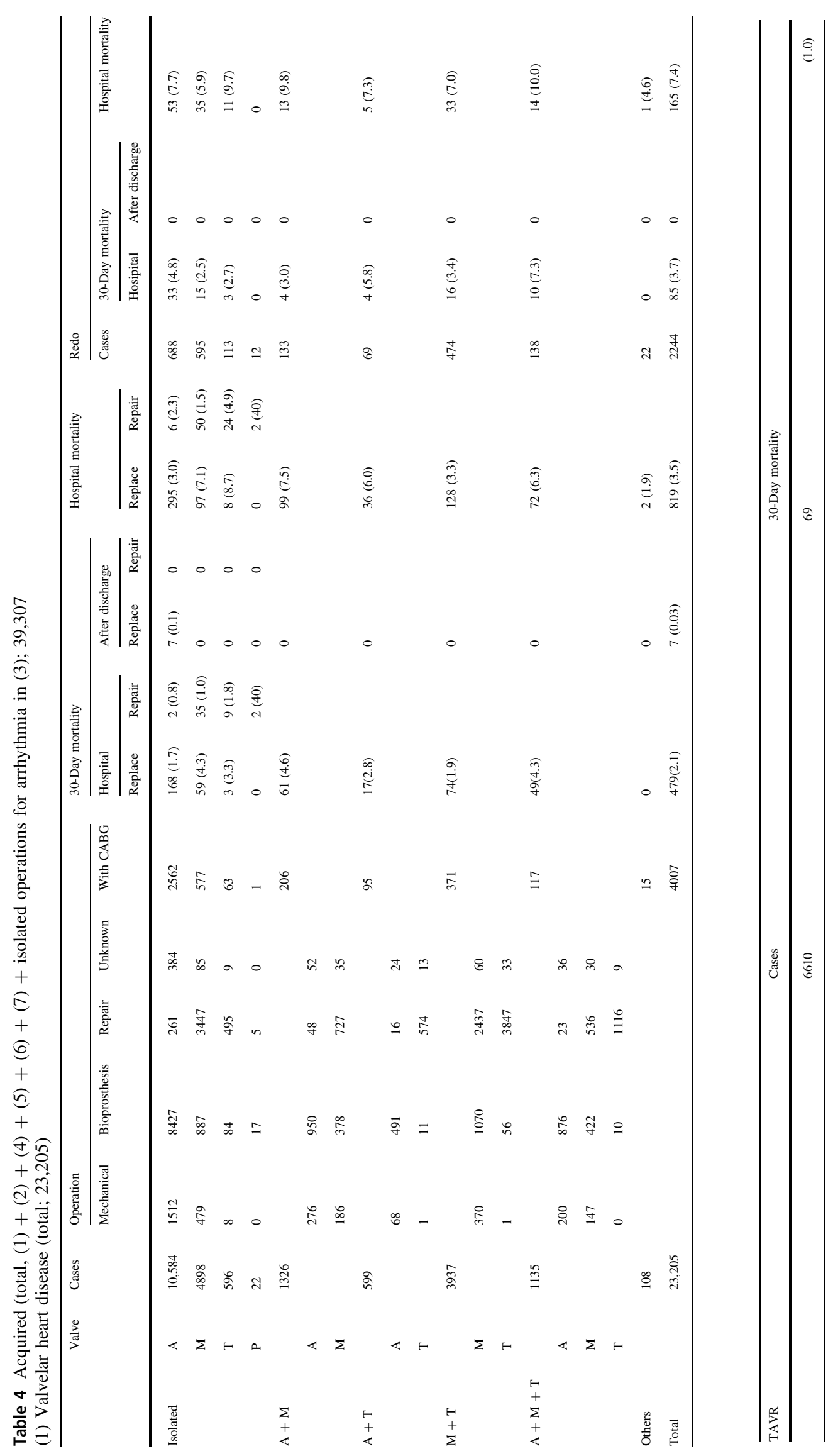




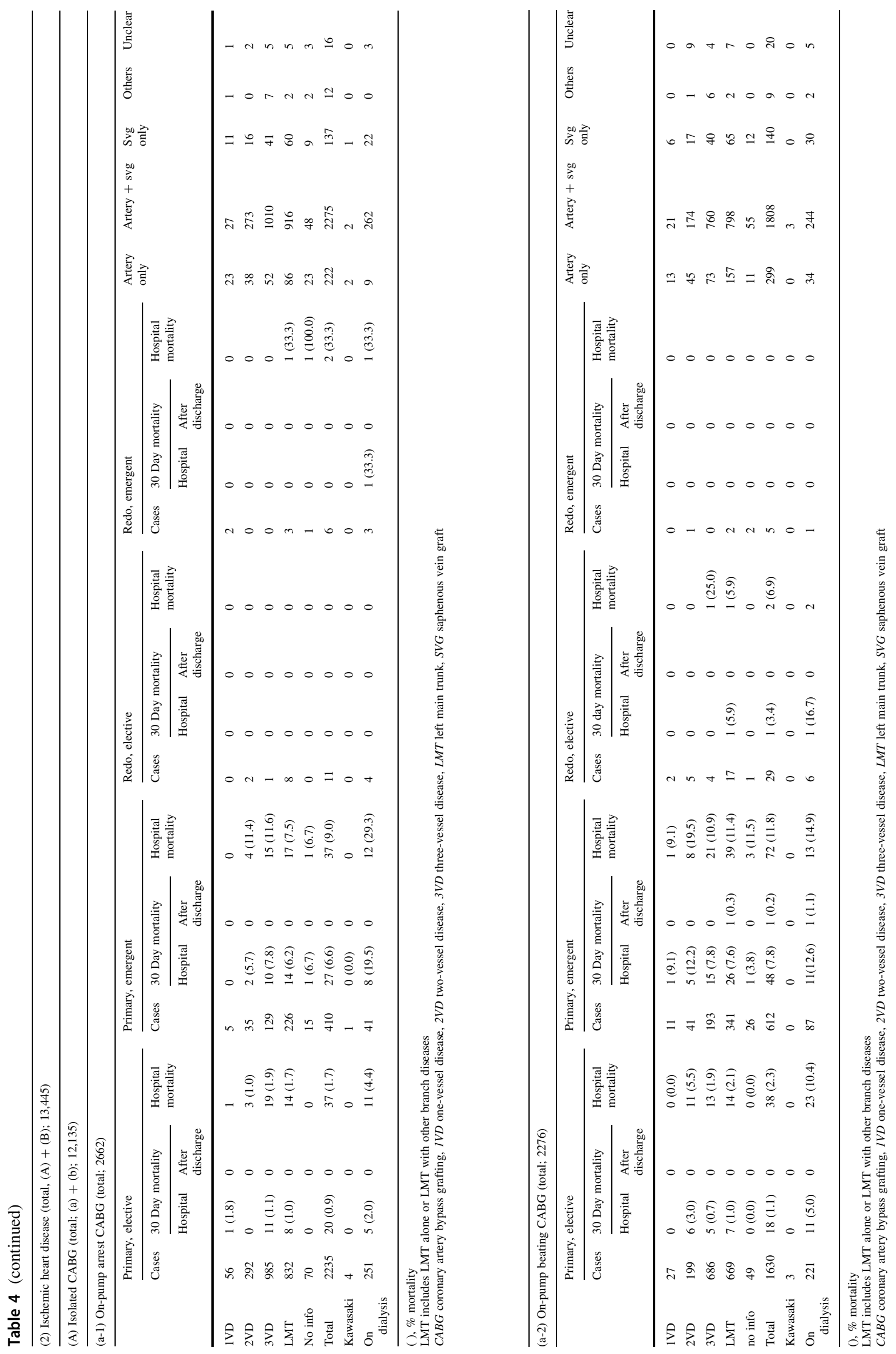




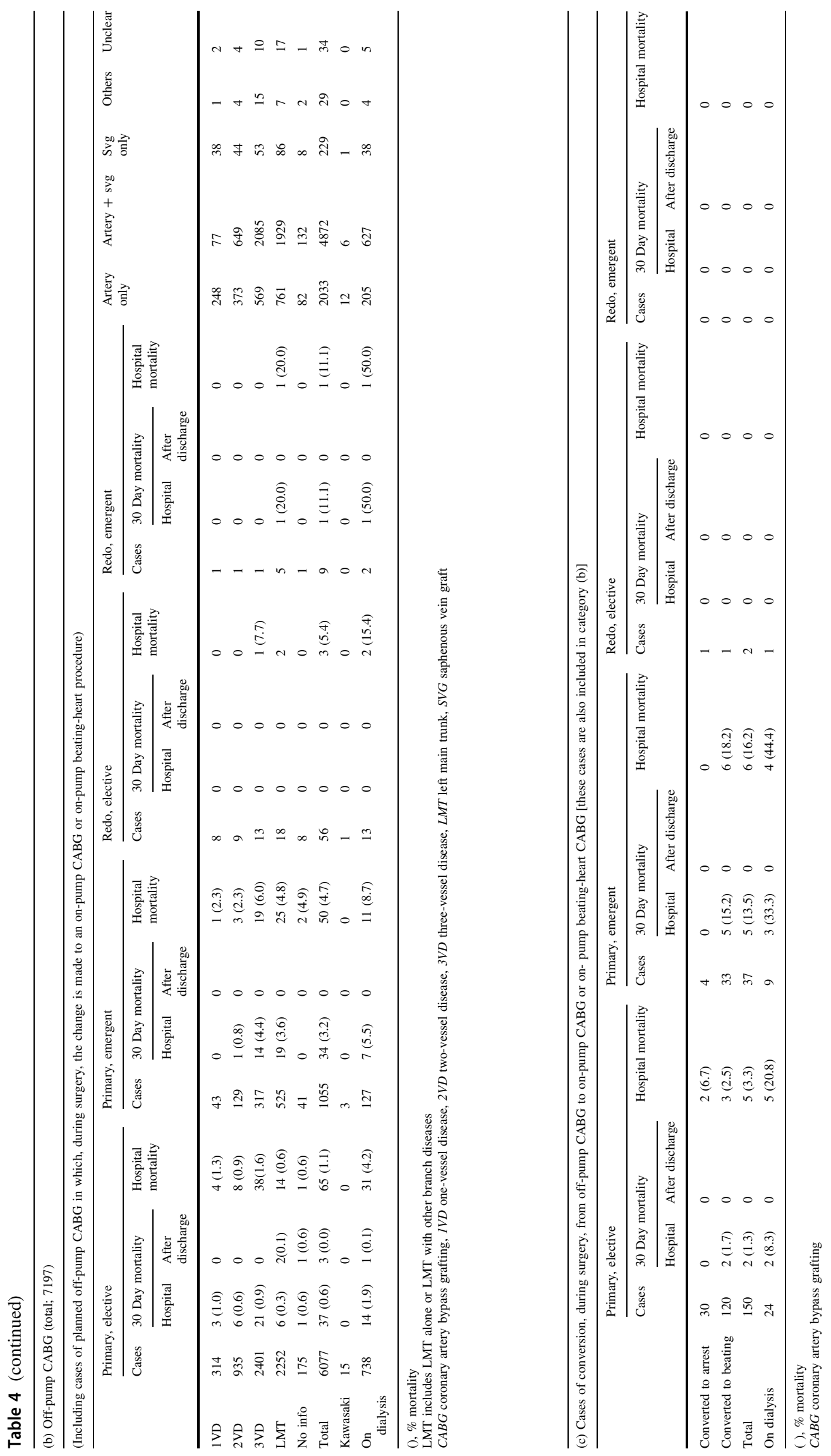



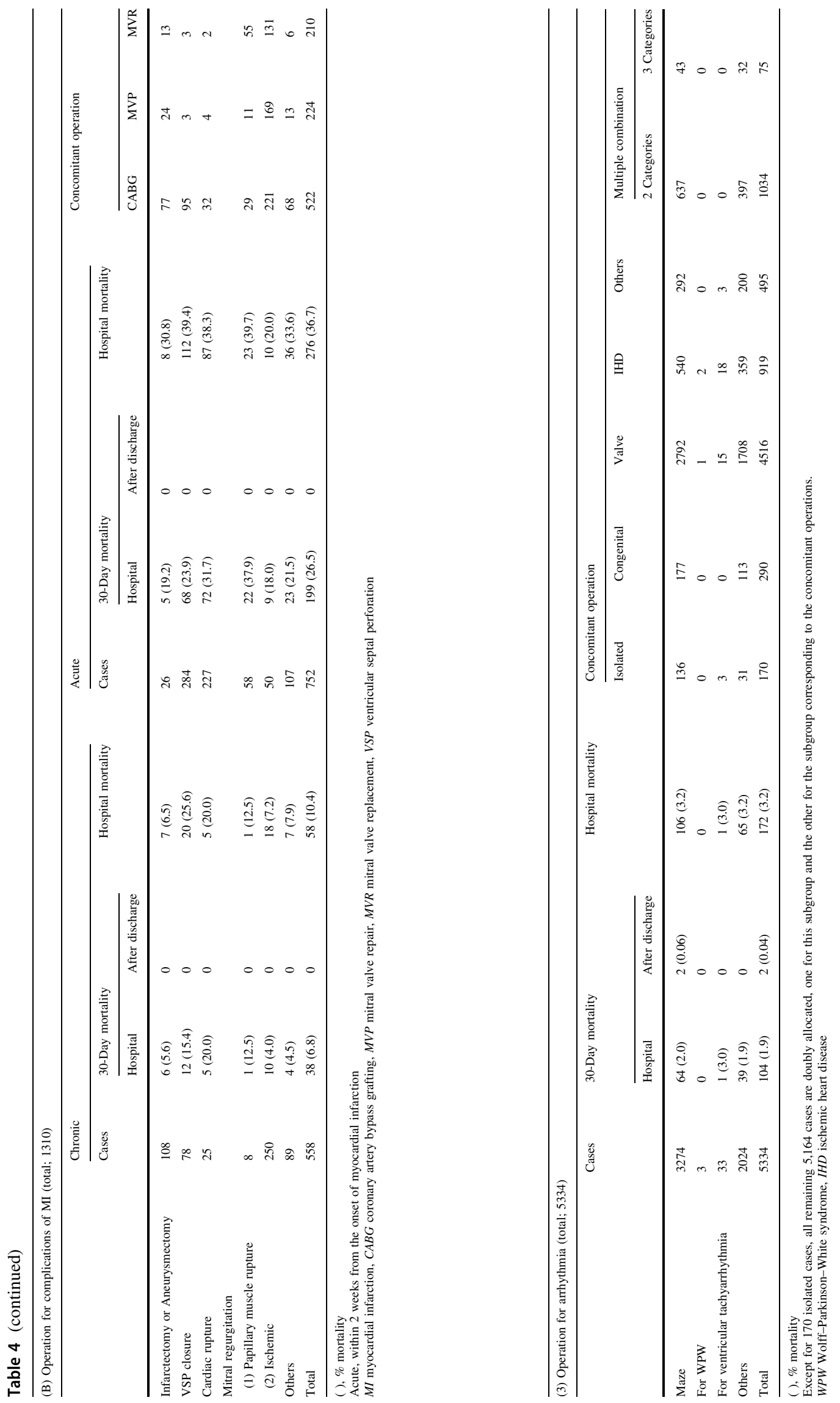


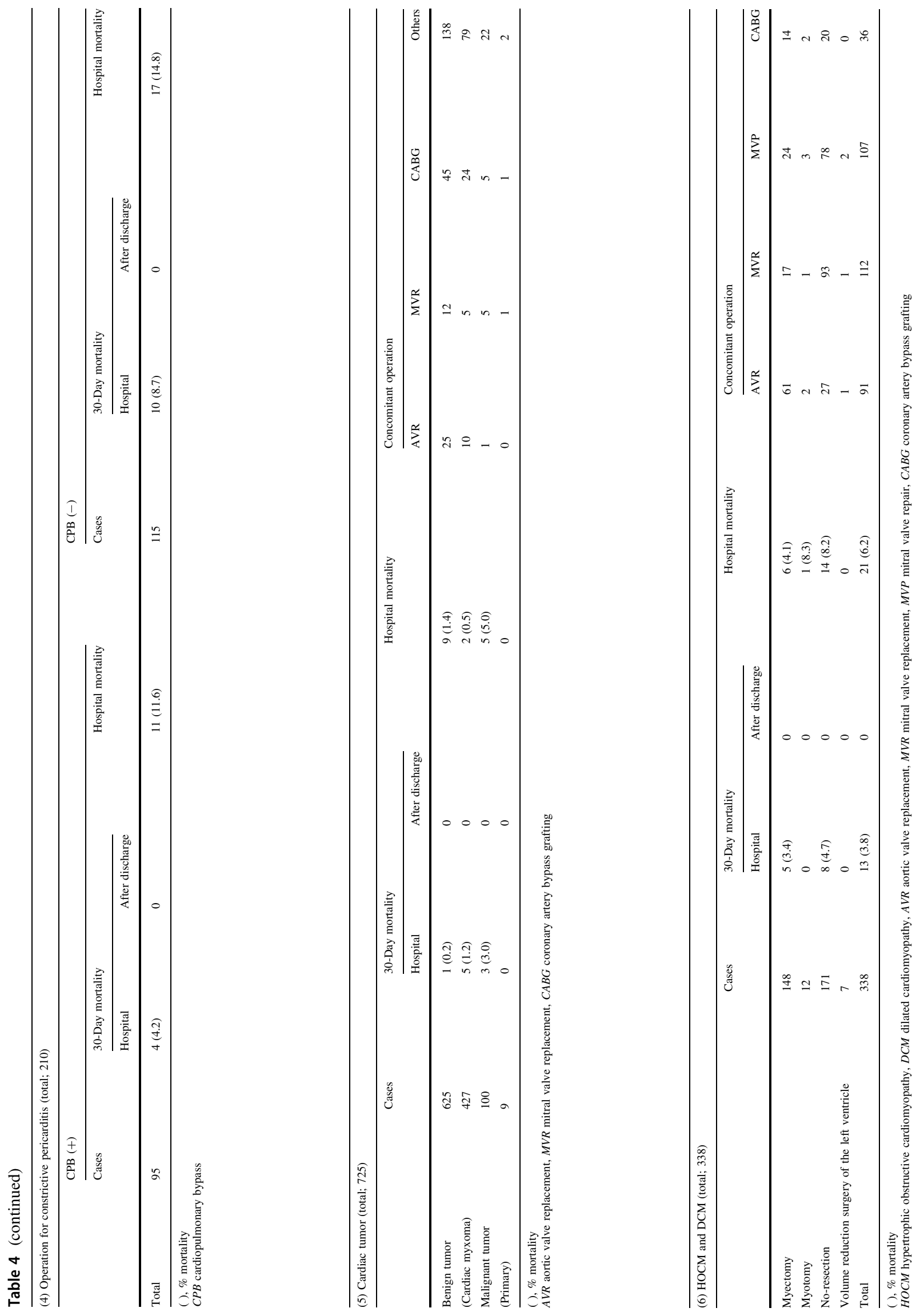




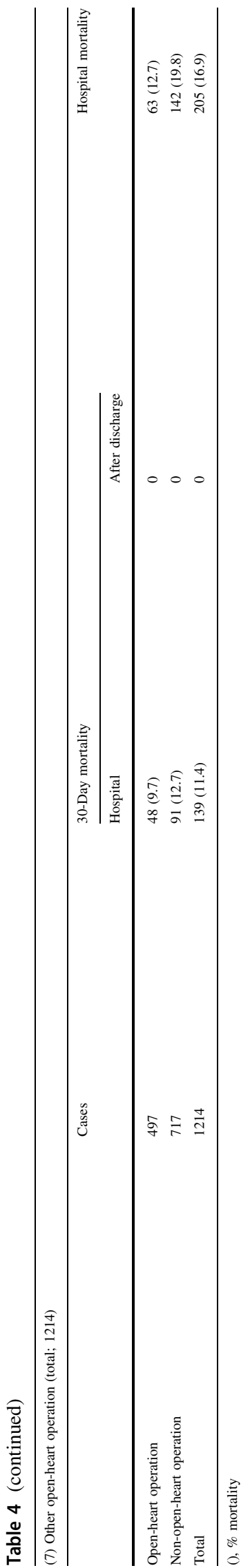

Among the 9253 procedures for congenital heart disease conducted in 2018, 7130 were open-heart surgeries, with an overall hospital mortality rate of $2.1 \%$. The number of surgeries for neonates and infants in 2018 did not differ significantly compared to that in 2008; however, hospital mortality improved from 10.8 to $8.3 \%$ for neonates and from 3.8 to $2.4 \%$ for infants. In 2018 , atrial septal defect was the most common disease (1402 cases), with patients aged 18 or older accounting for $58.6 \%$ of atrial septal defect surgery. Ventricular septal defect (perimembranous/muscular), which had been the most common disease in 2015 and 2016, was the second most common disease (1114 cases).

Within the past 10 years, hospital mortality for complex congenital heart disease was as follows (2008 [2], 2013 [3], and 2018): complete atrio-ventricular septal defect (3.5\%, $0.6 \%$, and $2.5 \%$, respectively); tetralogy of Fallot (1.8\%, $1.4 \%$, and $1.1 \%$, respectively); transposition of the great arteries with intact septum $(3.8 \%, 3.6 \%$, and $2.1 \%$, respectively), ventricular septal defect $(5.5 \%, 5.2 \%$, and $6.9 \%$, respectively), and single ventricle $(5.5 \%, 5.7 \%$, and $5.1 \%$, respectively); and hypoplastic left heart syndrome $(12.9 \%, 9.1 \%$, and $8.8 \%$, respectively). Currently, right heart bypass surgery has been commonly performed (359 bidirectional Glenn procedures excluding 36 DamusKaye-Stansel procedures and 395 Fontan-type procedures including total cavopulmonary connection) with acceptable hospital mortality rates $(2.2 \%$ and $1.5 \%)$. The Norwood type I procedure was performed in 130 cases, with a relatively low hospital mortality rate (15.4\%).

The total number of valvular heart disease procedures, excluding transcatheter procedures, was slightly lower than that in the previous year. Moreover, the number of isolated aortic valve replacement/repair with/without coronary artery bypass grafting $(\mathrm{CABG})(\mathrm{n}=10,584)$ was $1.0 \%$ lower than that in the previous year $(\mathrm{n}=10,690)$ but $2.0 \%$ higher than that 5 years ago $(n=10,379)$, despite the rapid utilization of transcatheter aortic valve replacement ( $\mathrm{n}=6610$ in 2018). The number of isolated mitral valve replacement/repair with/without CABG $(\mathrm{n}=4898)$ was $4.5 \%$ higher than that in the previous year $(n=4687)$ and $2.2 \%$ higher than that 5 years ago $(n=4793)$. A total of 10,744 and 2757 cases underwent aortic and mitral valve replacement with bioprosthesis, respectively. The rate at which bioprosthesis was utilized had increased dramatically from $30 \%$ in the early 2000 s $[4,5]$ to $83.9 \%$ and $70.0 \%$ in 2018 for aortic and mitral positions, respectively. Additionally, CABG was performed as a concomitant procedure in $17.3 \%$ of all valvular procedures $(16.7 \%$ in 2008 [2] and $17.8 \%$ in 2013 [3]). Valve repair had been popular for mitral and tricuspid valve positions (7147 and 6032 cases, respectively), but had been less frequently observed for aortic valve positions (348 patients, only $2.6 \%$ 
General Thoracic and Cardiovascular Surgery (2021) 69:179-212

193

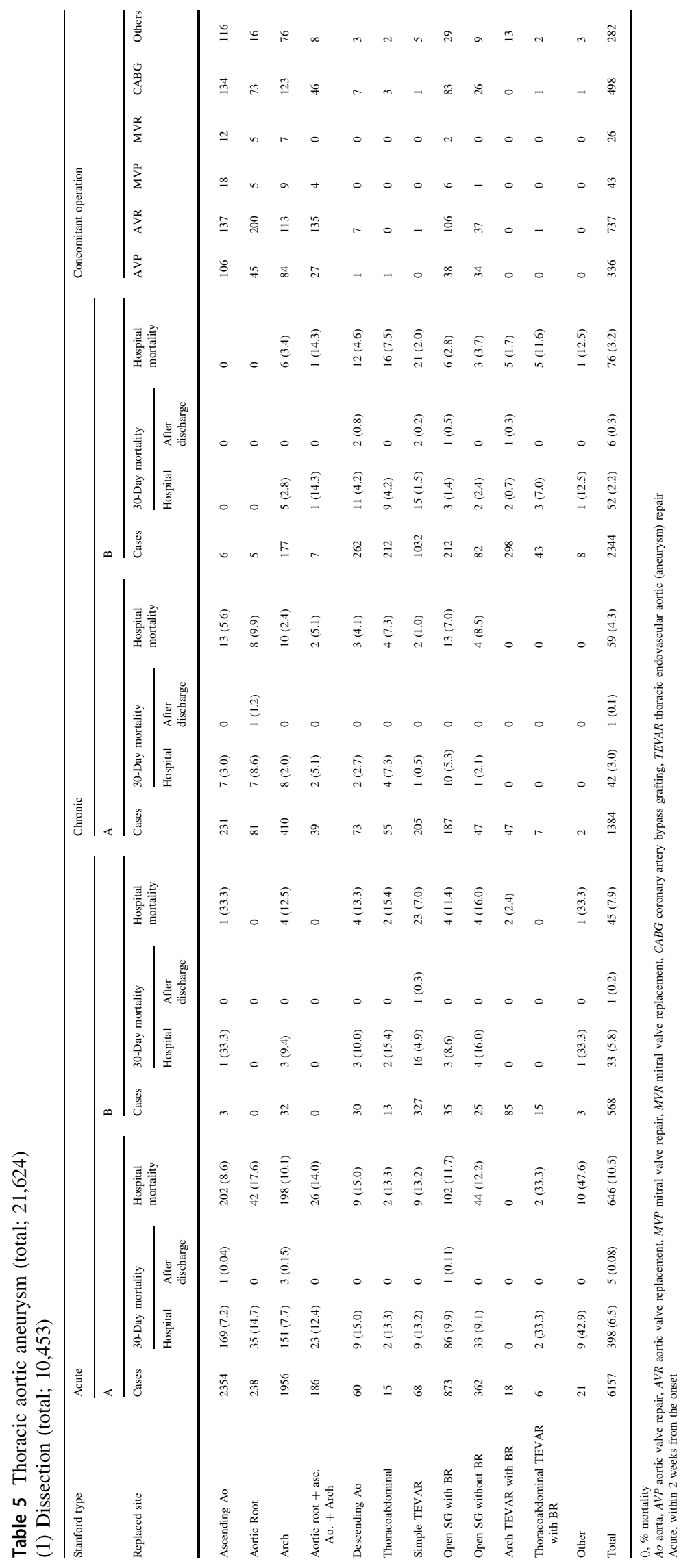

Springer 


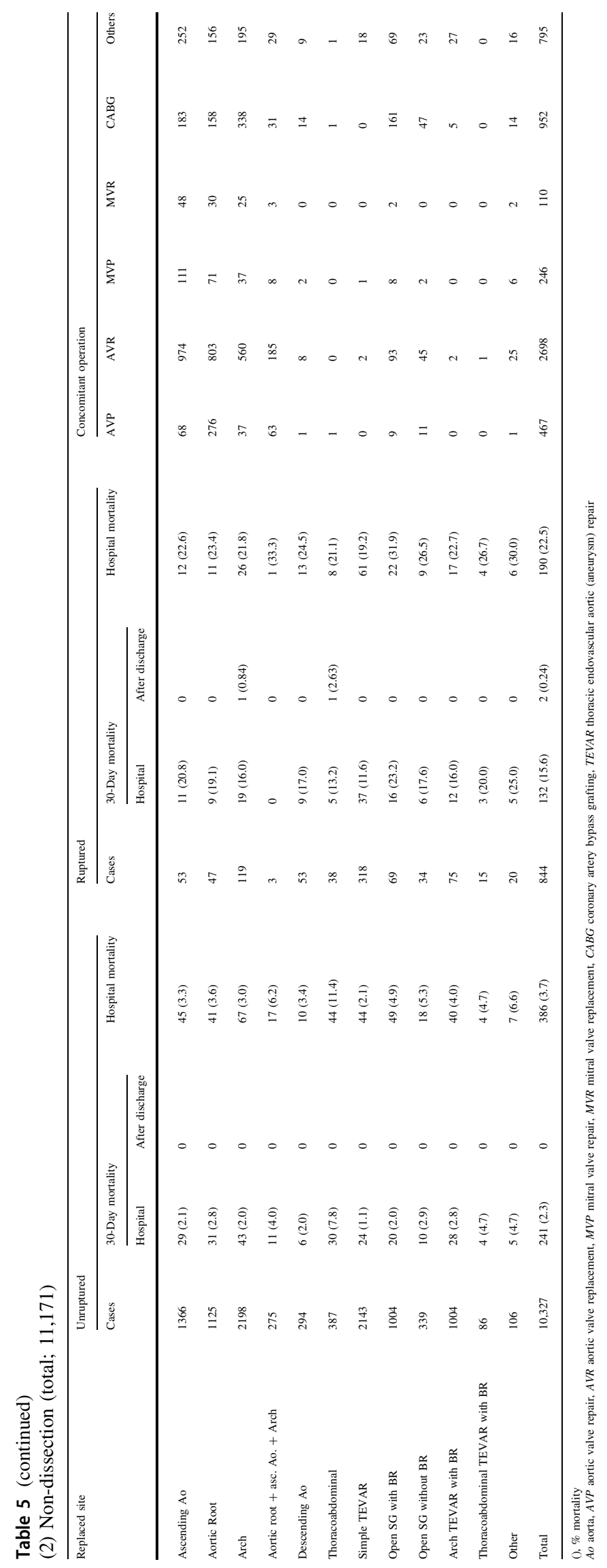


Table 6 Pulmonary thromboembolism (total; 138)

\begin{tabular}{lllll}
\hline & Cases & \multicolumn{2}{l}{30 -Day mortality } & Hospital mortality \\
\cline { 3 - 4 } & & Hospital & After discharge & \\
\hline Acute & 90 & $10(11.1)$ & 11 \\
Chronic & 48 & $2(4.2)$ & 3 \\
Total & 138 & $12(8.7)$ & 0 & $14(10.1)$ \\
\hline
\end{tabular}

(), \% mortality

Table 7 Implantation of VAD (total; 164)

\begin{tabular}{lllll}
\hline & Cases & 30-Day mortality & $\begin{array}{l}\text { Hospital } \\
\text { mortality }\end{array}$ \\
\cline { 3 - 4 } & & Hospital & $\begin{array}{l}\text { After } \\
\text { discharge }\end{array}$ & \\
\hline $\begin{array}{l}\text { Implantation of } \\
\text { VAD }\end{array}$ & 164 & $3(1.8)$ & $3(1.8)$ & $31(18.9)$ \\
\hline
\end{tabular}

(), \% mortality

$V A D$ ventricular assist devise

Table 8 Heart transplantation (total; 51)

\begin{tabular}{lllll}
\hline & Cases & \multicolumn{2}{l}{ 30-Day mortality } & $\begin{array}{l}\text { Hospital } \\
\text { mortality }\end{array}$ \\
\cline { 3 - 4 } & & Hospital & $\begin{array}{l}\text { After } \\
\text { discharge }\end{array}$ \\
\hline $\begin{array}{l}\text { Heart Transplantation } \\
\begin{array}{l}\text { Heart and Lung } \\
\quad\end{array}\end{array}$ & 51 & $1(2.0)$ & 0 & $2(3.9)$ \\
$\begin{array}{l}\text { Transplantation } \\
\text { Total }\end{array}$ & 51 & $1(2.0)$ & 0 & 0 \\
\hline
\end{tabular}

(), \% mortality

of all aortic valve procedures). Mitral valve repair constituted $63.3 \%$ of all mitral valve procedures. Hospital mortality rates for single valve replacement were $3.0 \%$ and $7.1 \%$ for aortic and mitral positions, respectively, but only $1.5 \%$ for mitral valve repair. Moreover, hospital mortality rates for redo valve surgery were $7.7 \%$ and $5.9 \%$ for the aortic and mitral positions, respectively. Finally, overall hospital mortality rates did not improve over the past 10 years (3.3\% in 2008 [2], 3.1\% in 2013 [3], and $3.5 \%$ in 2018).
Isolated $\mathrm{CABG}$ had been performed in 12,135 cases, accounting for only $68.3 \%$ of the number performed 10 years ago $(\mathrm{n}=17,764)[2]$. Among the aforementioned cases, $7197(58.8 \%)$ underwent off-pump CABG, with a success rate of $97.4 \%$. The percentage of intended offpump CABG in 2018 was similar to that in 2017 when it fell below $60 \%$ for the first time since 2004 [4]. Hospital mortality associated with primary elective $\mathrm{CABG}$ procedures among 7707 cases was $1.3 \%$, which did not differ from that in $2008(1.5 \%)$ [2]. Nonetheless, hospital mortality for primary emergency CABG among 1667 cases still remained high $(7.3 \%)$. The percentage of conversion from off-pump to on-pump CABG or on-pump beating-heart CABG was $2.6 \%$, with a hospital mortality rate of $5.8 \%$. Patients with end-stage renal failure on dialysis had higher hospital mortality rates than overall mortality, regardless of surgical procedure (on-pump arrest, on-pump beating, and off-pump). In this report, concomitant CABGs alongside other major procedures were not included under the ischemic heart disease category but rather under other categories, such as valvular heart disease and thoracic aortic aneurysm. Accordingly, the overall number of CABGs in 2018, including concomitant CABG with other major procedures, was 17,678 .

Measures for arrhythmia were performed primarily as concomitant procedures in 5334 cases, with a hospital mortality rate of $3.2 \%$. Pacemaker and implantable cardioverter-defibrillator implantation was not included in this category.

In 2018, 21,624 procedures for thoracic and thoracoabdominal aortae diseases were performed, among which 10,453 and 11,171 were for aortic dissection and non-dissection, respectively. The number of surgeries for aortic dissection this year was $3.6 \%$ higher than that in the preceding year $(n=10,086)$. Hospital mortality rates for the 6157 Stanford type A acute aortic dissections remained high $(10.5 \%)$. The number of procedures for non-dissected aneurysm increased by $4.8 \%$, with a hospital mortality rate of $5.2 \%$ for all aneurysms and $3.7 \%$ and $22.5 \%$ for unruptured and ruptured aneurysms, respectively. The rate at which thoracic endovascular aortic repair (TEVAR) has been performed for aortic diseases has been increasing. A total of 3974 patients with aortic dissection underwent stent graft placement: 2151 TEVARs and 1823 open stent graftings, respectively. Moreover, 1373 and 294 cases underwent TEVAR and open stent grafting for type B chronic aortic dissection, accounting for $58.6 \%$ and $12.5 \%$ of the total number of cases, respectively. Hospital 
mortality rates associated with simple TEVAR for type B aortic dissection were $7.0 \%$ and $2.0 \%$ for acute and chronic cases, respectively. A total of 5087 patients with non-dissected aortic aneurysm underwent stent graft placement, among which 3641 were TEVARs (a $10.6 \%$ increase compared to that in $2017, \mathrm{n}=3292$ ) and 1446 were open stent graftings (a 6.0\% increase compared to that in 2017, $n=1364$ ). Hospital mortality rates for TEVARs were $2.7 \%$ and $20.4 \%$ for unruptured and ruptured aneurysms, respectively, whereas those for open stenting were 5.0\% and $30.1 \%$ for unruptured and ruptured aneurysms, respectively.

\section{(B) General thoracic surgery}

The 2018 survey of general thoracic surgeries comprised 749 surgical units, with the bulk of the data submitted via a web-based collection system established by the NCD [1]. In total, 86,589 procedures had been reported by general thoracic surgery departments in 2018 , twice the number of surgeries compared to 2000 and approximately 11,200 more procedures than that in 2013 (Fig. 2).

In $2018,44,859$ procedures for primary lung cancer had been performed, a number that has continued to increase annually. Accordingly, the number of procedures in 2018 was 2.4 times higher than that in 2000 , with lung cancer procedures accounting for $52 \%$ of all general thoracic surgeries (Table 9).

Information regarding the number of video-assisted thoracoscopic surgery (VATS), defined as surgical procedures utilizing a skin incision over $8 \mathrm{~cm}$ and/or a minithoracotomy (hybrid) approach, has been available since the 2015 annual report. The number of VATS procedures for benign pulmonary tumors and primary lung cancer and the total number of VATS procedures in 2016
Table 9 Total cases of general thoracic surgery during 2018

\begin{tabular}{lll}
\hline & Cases & $\%$ \\
\hline Benign pulmonary tumor & 2342 & 2.7 \\
Primary lung cancer & 44,859 & 51.8 \\
Other primary malignant pulmonary tumor & 384 & 0.4 \\
Metastatic pulmonary tumor & 8978 & 10.4 \\
Tracheal tumor & 127 & 0.1 \\
Mesothelioma & 664 & 0.8 \\
Chest wall tumor & 656 & 0.8 \\
Mediastinal tumor & 5361 & 6.2 \\
Thymectomy for MG without thymoma & 151 & 0.2 \\
Inflammatory pulmonary disease & 2400 & 2.8 \\
Empyema & 3103 & 3.6 \\
Bullous disease excluding pneumothorax & 376 & 0.4 \\
Pneumothorax & 14,731 & 17.0 \\
Chest wall deformity & 176 & 0.2 \\
Diaphragmatic hernia including traumatic & 30 & 0.0 \\
Chest trauma excluding diaphragmatic hernia & 431 & 0.5 \\
Lung transplantation & 71 & 0.1 \\
Others & 1749 & 2.0 \\
Total & 86,589 & 100.0 \\
\hline & &
\end{tabular}

are presented in Tables 10, 11, 13, 16, 17, 18, 19, 20, 21, $22,23,25,26,27$, respectively.

In 2018, a total of 2342 procedures for benign pulmonary tumors had been conducted (Table 10). Hamartomas were the most frequent benign pulmonary tumors diagnosed, with 2222 patients (95\%) undergoing VATS.

Additional information on primary malignant pulmonary tumors is shown in Tables 11, 12. Accordingly, adenocarcinoma had been the most frequently diagnosed lung cancer subtype ( $71 \%$ of all lung cancers), followed by

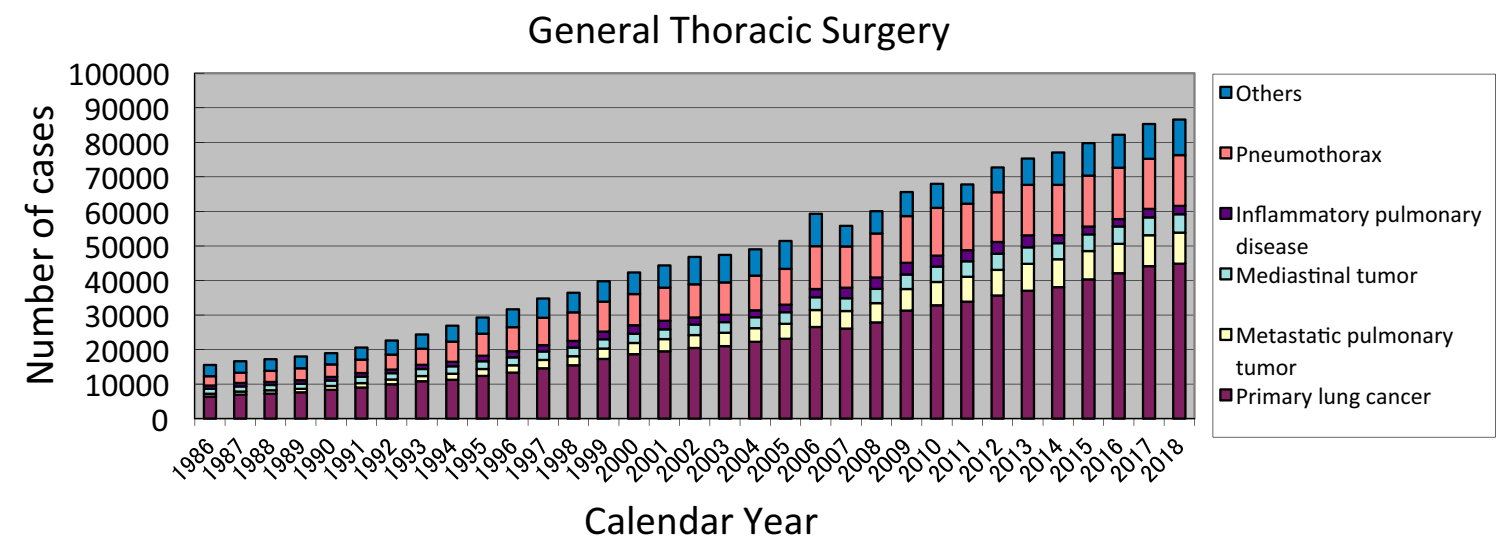

Fig. 2 General thoracic surgery 
Table 10 Benign pulmonary tumor

\begin{tabular}{|c|c|c|c|c|c|}
\hline & \multirow[t]{2}{*}{ Cases } & \multicolumn{2}{|c|}{ 30-Day mortality } & \multirow{2}{*}{$\begin{array}{l}\text { Hospital } \\
\text { mortality }\end{array}$} & \multirow{2}{*}{$\begin{array}{l}\text { By } \\
\text { VATS }\end{array}$} \\
\hline & & Hospital & $\begin{array}{l}\text { After } \\
\text { discharge }\end{array}$ & & \\
\hline \multicolumn{6}{|c|}{ Benign pulmonary tumor } \\
\hline Hamartoma & 527 & 0 & 0 & 0 & 503 \\
\hline $\begin{array}{l}\text { Sclerosing } \\
\text { hemangioma }\end{array}$ & 109 & 0 & 0 & 0 & 104 \\
\hline Papilloma & 23 & 0 & 0 & 0 & 22 \\
\hline $\begin{array}{l}\text { Mucous gland } \\
\text { adenoma } \\
\text { bronchial }\end{array}$ & 4 & 0 & 0 & 0 & 4 \\
\hline Fibroma & 136 & 0 & 0 & 0 & 123 \\
\hline Lipoma & 8 & 0 & 0 & 0 & 7 \\
\hline Neurogenic tumor & 18 & 0 & 0 & 0 & 15 \\
\hline Clear cell tumor & 2 & 0 & 0 & 0 & 2 \\
\hline Leiomyoma & 12 & 0 & 0 & 0 & 12 \\
\hline Chondroma & 4 & 0 & 0 & 0 & 4 \\
\hline $\begin{array}{l}\text { Inflammatory } \\
\text { myofibroblastic } \\
\text { tumor }\end{array}$ & 0 & 0 & 0 & 0 & 0 \\
\hline Pseudolymphoma & 26 & 0 & 0 & 0 & 25 \\
\hline Histiocytosis & 12 & 0 & 0 & 0 & 12 \\
\hline Teratoma & 7 & 0 & 0 & 0 & 6 \\
\hline Others & 1454 & $1(0.1)$ & $1(0.1)$ & $1(0.1)$ & 1383 \\
\hline Total & 2342 & $1(0.04)$ & $1(0.04)$ & $1(0.04)$ & 2222 \\
\hline
\end{tabular}

(), mortality $\%$

squamous cell carcinoma (18\%). Sublobar resection was performed in 12,819 lung cancer cases (29\% of all cases) and lobectomy in 31,365 cases ( $70 \%$ of all cases). Sleeve lobectomy was performed in 474 cases, while pneumonectomy was required in 324 cases ( $0.7 \%$ of all cases). VATS lobectomy for lung cancer was performed in 22,880 cases (73\% of all lobectomy cases). The number of patients aged 80 years or older who underwent lung cancer surgery was 6115 (14\%). Among those who died within 30 days following surgery, 107 died prior to hospital discharge, while 28 died after discharge. Overall, 135 patients died within 30 days after surgery (30-day mortality rate, $0.3 \%$ ), while 242 died prior to discharge (hospital mortality rate, $0.5 \%)$. Moreover, 30-day mortality rates according to procedure were $0.1 \%, 0.2 \%$, and $1.5 \%$ for segmentectomy, lobectomy, and pneumonectomy, respectively. Interstitial pneumonia had been the leading cause of death following lung cancer surgery, followed by pneumonia, cardiovascular events, and respiratory failure.

The procedures for metastatic pulmonary tumors, 8978 of which were performed in 2018, are shown in Table 13. Among such procedures, colorectal cancer had been the most frequent diagnosis (49\% of all cases).
Table 11 Primary malignant pulmonary tumor

\begin{tabular}{|c|c|c|c|c|c|}
\hline & \multirow[t]{2}{*}{ Cases } & \multicolumn{2}{|c|}{ 30-Day mortality } & \multirow{2}{*}{$\begin{array}{l}\text { Hospital } \\
\text { mortality }\end{array}$} & \multirow[t]{2}{*}{ VATS } \\
\hline & & Hospital & $\begin{array}{l}\text { After } \\
\text { discharge }\end{array}$ & & \\
\hline $\begin{array}{l}\text { Primary malignant pulmonary } \\
\text { tumor }\end{array}$ & 45,243 & $107(0.2)$ & $28(0.1)$ & $244(0.5)$ & 34,249 \\
\hline Lung cancer & 44,859 & $107(0.2)$ & $28(0.1)$ & $242(0.5)$ & 34,249 \\
\hline Adenocarcinoma & 31,720 & $52(0.2)$ & $11(0.03)$ & $92(0.3)$ & \\
\hline Squamous cell carcinoma & 8265 & $40(0.5)$ & $13(0.2)$ & $106(1.3)$ & \\
\hline Large cell carcinoma & 280 & 0 & 0 & $1(0.4)$ & \\
\hline LCNEC & 543 & $2(0.4)$ & 0 & $4(0.7)$ & \\
\hline Small cell carcinoma & 785 & $3(0.4)$ & $2(0.3)$ & $10(1.3)$ & \\
\hline Adenosquamous carcinoma & 560 & $2(0.4)$ & 0 & $\begin{array}{l}4 \\
(0.7)\end{array}$ & \\
\hline $\begin{array}{l}\text { Carcinoma with pleomorphic, } \\
\text { sarcomatoid or } \\
\text { sarcomatous elements }\end{array}$ & 511 & $4(0.8)$ & 0 & $10(2.0)$ & \\
\hline Carcinoid & 252 & 0 & 0 & 0 & \\
\hline $\begin{array}{l}\text { Carcinomas of salivary-gland } \\
\text { type }\end{array}$ & 40 & 0 & 0 & $1(2.5)$ & \\
\hline Unclassified & 46 & 0 & 0 & 0 & \\
\hline Multiple lung cancer & 1554 & $2(0.1)$ & $2(0.1)$ & $9(0.6)$ & \\
\hline Others & 302 & $2(0.7)$ & 0 & $\begin{array}{l}5 \\
(1.7)\end{array}$ & \\
\hline Wedge resection & 7683 & $11(0.1)$ & $13(0.2)$ & $21(0.3)$ & 6900 \\
\hline Segmental excision & 5136 & $5(0.1)$ & $1(0.02)$ & $17(0.3)$ & 4219 \\
\hline (Sleeve segmental excision) & 12 & 0 & 0 & 0 & 6 \\
\hline Lobectomy & 31,365 & $83(0.3)$ & $14(0.04)$ & $188(0.6)$ & 22,880 \\
\hline (Sleeve lobectomy) & 474 & $1(0.2)$ & 0 & $3(0.6)$ & 75 \\
\hline Pneumonectomy & 324 & $5(1.5)$ & 0 & $10(3.1)$ & 42 \\
\hline (Sleeve pneumonectomy) & 9 & 0 & 0 & 0 & 0 \\
\hline Other bronchoplasty & 34 & $1(2.9)$ & 0 & $1(2.9)$ & 7 \\
\hline Pleuropneumonectomy & 2 & 0 & 0 & 0 & 0 \\
\hline Others & 315 & $2(0.6)$ & 0 & $5(1.6)$ & 201 \\
\hline Unknown & 0 & 0 & 0 & 0 & \\
\hline Sarcoma & 51 & 0 & 0 & $1(2.0)$ & \\
\hline $\mathrm{AAH}$ & 103 & 0 & 0 & 0 & \\
\hline Others & 230 & 0 & 0 & $1(0.4)$ & \\
\hline
\end{tabular}

(), mortality \%

A total of 59 procedures for malignant tracheal tumor were performed in 2018; however, 30 patients underwent sleeve resection and reconstruction (Table 14).

Overall, 664 pleural tumors had been diagnosed in 2018 (Table 15), with diffuse malignant pleural mesothelioma being the most frequent histologic diagnosis. Total pleurectomy was performed in 100 cases and extrapleural pneumonectomy in 64 cases. The 30-day mortality rate was $1 \%$ and $3 \%$ following total pleurectomy and extrapleural pneumonectomy, respectively, both of which had better outcomes than previously reported. 
Table 12 Details of lung cancer operations

\begin{tabular}{|c|c|}
\hline \multicolumn{2}{|l|}{ TNM } \\
\hline c-Stage & Cases \\
\hline IA 1 & 7,832 \\
\hline IA2 & 12,773 \\
\hline IA3 & 8,048 \\
\hline IB & 4,977 \\
\hline IIA & 1,577 \\
\hline IIB & 3,862 \\
\hline IIIA & 2,683 \\
\hline IIIB & 499 \\
\hline IIIC & 26 \\
\hline IVA & 388 \\
\hline IVB & 81 \\
\hline NA & 2,113 \\
\hline Total & 44,859 \\
\hline Sex & Cases \\
\hline Male & 27,385 \\
\hline Female & 17,474 \\
\hline NA & 0 \\
\hline Total & 44,859 \\
\hline Cause of death & Cases \\
\hline Cardiovascular & 24 \\
\hline Pneumonia & 41 \\
\hline Pyothorax & 2 \\
\hline Bronchopleural fistula & 13 \\
\hline Respiratory failure & 22 \\
\hline Pulmonary embolism & 5 \\
\hline Interstitial pneumonia & 79 \\
\hline Brain infarction or bleeding & 13 \\
\hline Others & 65 \\
\hline Unknown & 6 \\
\hline Total & 270 \\
\hline p-Stage & Cases \\
\hline 0 (pCR) & 3,234 \\
\hline IA 1 & 9,035 \\
\hline IA2 & 9,839 \\
\hline IA3 & 4,890 \\
\hline IB & 6,107 \\
\hline IIA & 1,190 \\
\hline IIB & 4,561 \\
\hline IIIA & 3,808 \\
\hline IIIB & 820 \\
\hline IIIC & 16 \\
\hline IVA & 1,010 \\
\hline IVB & 73 \\
\hline NA & 276 \\
\hline
\end{tabular}

Table 12 (continued)

\begin{tabular}{ll}
\hline p-Stage & Cases \\
\hline Total & 44,859 \\
\hline Age (years) & Cases \\
\hline$<20$ & 25 \\
$20-29$ & 31 \\
$30-39$ & 277 \\
$40-49$ & 1,195 \\
$50-59$ & 3,736 \\
$60-69$ & 13,290 \\
$70-79$ & 20,190 \\
$80-89$ & 6,003 \\
$\geq 90$ & 112 \\
NA & 0 \\
Total & 44,859 \\
\hline
\end{tabular}

Overall, 656 chest wall tumor resections had been performed in 2018 (Table 16), among which 345 (53\%) were benign. Among the 311 malignant chest wall tumors, 179 $(58 \%)$ were metastatic.

A total of 5361 mediastinal tumors were resected in 2018, a slight increase compared to that in the previous year (Table 17). Thymic epithelial tumors-including 2098 thymomas, 325 thymic carcinomas, and 43 thymic carcinoids-were the most frequently diagnosed mediastinal tumor subtype in 2018.

In total, 499 patients underwent thymectomy for myasthenia gravis (Table 18), among which 348 procedures were associated with thymoma.

Overall, 22,996 patients underwent procedures for nonneoplastic disease. Accordingly, 2400 patients underwent lung resection for inflammatory lung diseases (Table 19), among which $22 \%$ and $14 \%$ were associated with mycobacterial infections and fungal infections, respectively. Procedures for inflammatory nodules were performed in cases where lung cancer was suspected prior to surgery (902 cases, $38 \%$ ).

A total of 3103 procedures were performed for empyema (Table 20), among which $2402(77 \%)$ were acute and 701 were chronic. Moreover, 509 patients with acute empyema and 325 patients with chronic empyema had developed bronchopleural fistulas. The hospital mortality rate was $13 \%$ among patients with acute empyema with fistula.

In 2018, 106 operations were performed for descending necrotizing mediastinitis (Table 21), with a hospital mortality rate of $6 \%$. Furthermore, 376 procedures were 
Table 13 Metastatic pulmonary tumor

\begin{tabular}{llllll}
\hline & Cases & \multicolumn{2}{l}{30 -Day mortality } & Hospital mortality & \multirow{2}{*}{ VATS } \\
\cline { 3 - 4 } & & Hospital & After discharge & \\
\hline Metastatic pulmonary tumor & 8978 & $6(0.1)$ & $4(0.04)$ & $13(0.1)$ & 8342 \\
Colorectal & 4396 & $2(0.05)$ & $1(0.02)$ & $5(0.1)$ & 4088 \\
Hepatobiliary/Pancreatic & 433 & 0 & 0 & 0 & 414 \\
Uterine & 504 & 0 & $1(0.2)$ & 0 & 469 \\
Mammary & 543 & $2(0.4)$ & 0 & $3(0.6)$ & 522 \\
Ovarian & 82 & 0 & 0 & 0 & 76 \\
Testicular & 60 & 0 & 0 & 0 & 56 \\
Renal & 690 & 0 & 0 & 0 & 646 \\
Skeletal & 110 & 0 & 0 & 0 & 96 \\
Soft tissue & 261 & 0 & 0 & 0 & 238 \\
Otorhinolaryngological & 471 & 0 & $1(0.2)$ & 0 & 442 \\
Pulmonary & 470 & $1(0.2)$ & 0 & $2(0.4)$ & 405 \\
Others & 958 & $1(0.1)$ & $1(0.1)$ & $3(0.3)$ & 890 \\
\hline
\end{tabular}

(), mortality \%

Table 14 Tracheal tumor

\begin{tabular}{|c|c|c|c|c|}
\hline & \multirow[t]{2}{*}{ Cases } & \multicolumn{2}{|c|}{ 30-Day mortality } & \multirow{2}{*}{$\begin{array}{l}\text { Hospital } \\
\text { mortality }\end{array}$} \\
\hline & & Hospital & $\begin{array}{l}\text { After } \\
\text { discharge }\end{array}$ & \\
\hline Tracheal tumor & 127 & $5(3.9)$ & $1(0.8)$ & $6(4.7)$ \\
\hline \multicolumn{5}{|l|}{ A. Primary malignant tumor } \\
\hline \multicolumn{5}{|l|}{ Histological classification } \\
\hline Squamous cell carcinoma & 17 & $1(5.9)$ & 0 & $1(5.9)$ \\
\hline Adenoid cystic carcinoma & 17 & 0 & 0 & 0 \\
\hline Mucoepidermoid carcinoma & 6 & 0 & 0 & 0 \\
\hline Others & 19 & 0 & 0 & $1(5.3)$ \\
\hline Total & 59 & $1(1.7)$ & 0 & $2(3.4)$ \\
\hline $\begin{array}{l}\text { B. Metastatic/invasive malignant tumor, e.g. invasion of } \\
\text { thyroid cancer }\end{array}$ & 33 & $1(3.0)$ & $1(3.0)$ & $1(3.0)$ \\
\hline \multicolumn{5}{|l|}{ C. Benign tracheal tumor } \\
\hline \multicolumn{5}{|l|}{ Histological classification } \\
\hline Papilloma & 1 & 0 & 0 & 0 \\
\hline Adenoma & 2 & 0 & 0 & 0 \\
\hline Neurofibroma & 1 & 0 & 0 & 0 \\
\hline Chondroma & 0 & 0 & 0 & 0 \\
\hline Leiomyoma & 3 & 0 & 0 & 0 \\
\hline Others & 28 & $3(10.7)$ & 0 & $3(10.7)$ \\
\hline Histology unknown & 0 & 0 & 0 & 0 \\
\hline Total & 35 & $3(8.6)$ & 0 & $3(8.6)$ \\
\hline \multicolumn{5}{|l|}{ Operation } \\
\hline Sleeve resection with reconstruction & 30 & 0 & 0 & 0 \\
\hline Wedge with simple closure & 1 & 0 & 0 & 0 \\
\hline Wedge with patch closure & 1 & 0 & 0 & 0 \\
\hline Total laryngectomy with tracheostomy & 0 & 0 & 0 & 0 \\
\hline Others & 3 & 0 & 0 & 0 \\
\hline Unknown & 0 & 0 & 0 & 0 \\
\hline Total & 35 & 0 & 0 & 0 \\
\hline
\end{tabular}

(), mortality \% 
Table 15 Tumor of pleural origin

\begin{tabular}{|c|c|c|c|c|c|}
\hline \multirow{2}{*}{\multicolumn{2}{|c|}{ Histological classification }} & \multirow[t]{2}{*}{ Cases } & \multicolumn{2}{|c|}{ 30-Day mortality } & \multirow[t]{2}{*}{ Hospital mortality } \\
\hline & & & Hospital & After discharge & \\
\hline \multicolumn{2}{|l|}{ Solitary fibrous tumor } & 146 & 0 & 0 & 0 \\
\hline \multicolumn{2}{|c|}{ Diffuse malignant pleural mesothelioma } & 264 & $4(1.5)$ & $1(0.4)$ & $13(4.9)$ \\
\hline \multicolumn{2}{|c|}{ Localized malignant pleural mesothelioma } & 32 & 0 & 0 & $1(3.1)$ \\
\hline \multicolumn{2}{|l|}{ Others } & 222 & $1(0.5)$ & 0 & $4(1.8)$ \\
\hline \multicolumn{2}{|l|}{ Total } & 664 & $5(0.8)$ & $1(0.2)$ & $18(2.7)$ \\
\hline \multirow[t]{2}{*}{ Operative procedure } & \multirow[t]{2}{*}{ Cases } & \multicolumn{3}{|c|}{ 30-Day mortality } & Hospital mortality \\
\hline & & \multicolumn{2}{|c|}{ Hospital } & After discharge & \\
\hline Extrapleural pneumonectomy & 64 & \multicolumn{2}{|c|}{$2(3.1)$} & 0 & $6(9.4)$ \\
\hline Total pleurectomy & 100 & \multicolumn{2}{|c|}{$1(1.0)$} & 0 & $2(2.0)$ \\
\hline Others & 100 & \multicolumn{2}{|c|}{$1(1.0)$} & $1(1.0)$ & $5(5.0)$ \\
\hline Total & 264 & \multicolumn{2}{|c|}{$4(1.5)$} & $1(0.4)$ & $13(4.9)$ \\
\hline
\end{tabular}

(), mortality $\%$ conducted for bullous diseases (Table 22), while only 23 patients underwent lung volume reduction surgery.

A total of 14,731 procedures were performed for spontaneous pneumothorax (Table 23). Among the 11,124 procedures for primary pneumothorax, 2825 (25\%) were bullectomies alone, while $7632(69 \%)$ required additional procedures. A total of 3607 procedures for secondary pneumothorax were conducted, with COPD being the most prevalent associated disease (2437 cases, $68 \%$ ). The hospital mortality rate for secondary pneumothorax associated with COPD was $2.7 \%$.

The 2018 survey reported 176 procedures for chest wall deformity (Table 24). However, this may have been underestimated given that the Nuss procedure for pectus excavatum was more likely to have been performed in pediatric surgery centers not associated with the Japanese Association for Thoracic Surgery.
Overall, 30 patients underwent surgical treatment for diaphragmatic hernia (Table 25). This figure may have also been underestimated considering that procedures may have been classified as gastrointestinal surgery.

The survey reported 431 procedures for chest trauma, excluding iatrogenic injuries (Table 26), with a hospital mortality rate of $8 \%$.

Table 27 summarizes the procedures for other diseases, including 84 and 103 cases of arteriovenous malformation and pulmonary sequestration, respectively.

A total of 71 lung transplantations were performed in 2018 (Table 28), among which 57 and 14 were from braindead and living related donors, respectively.

The number of VATS procedures has continued to increase annually, ultimately reaching 71,171 ( $82 \%$ of all general thoracic surgeries) in 2018 (Table 29).

Table 16 Chest wall tumor

\begin{tabular}{llllll}
\hline & Cases & \multicolumn{2}{l}{30 -Day mortality } & Hospital mortality & VATS \\
\cline { 3 - 4 } & & Hospital & After discharge & \\
\hline Primary malignant tumor & 132 & 0 & 0 & 0 & 71 \\
Metastatic malignant tumor & 179 & $1(0.6)$ & 0 & $1(0.6)$ & 84 \\
Benign tumor & 345 & 0 & 0 & 0 & 265 \\
Total & 656 & $1(0.2)$ & 0 & $1(0.2)$ & 420 \\
\hline
\end{tabular}

(), mortality \% 
Table 17 Mediastinal tumor

\begin{tabular}{|c|c|c|c|c|c|}
\hline & \multirow[t]{2}{*}{ Cases } & \multicolumn{2}{|c|}{ 30-Day mortality } & \multirow[t]{2}{*}{ Hospital mortality } & \multirow[t]{2}{*}{ By VATS } \\
\hline & & Hospital & After discharge & & \\
\hline Mediastinal tumor & 5361 & $2(0.04)$ & $1(0.02)$ & $6(0.1)$ & 4009 \\
\hline Thymoma* & 2098 & 0 & 0 & $2(0.1)$ & 1379 \\
\hline Thymic cancer & 325 & $1(0.3)$ & 0 & $1(0.3)$ & 171 \\
\hline Thymus carcinoid & 43 & 0 & 0 & 0 & 22 \\
\hline Germ cell tumor & 81 & 0 & 0 & 0 & 44 \\
\hline Benign & 58 & 0 & 0 & 0 & 35 \\
\hline Malignant & 23 & 0 & 0 & 0 & 9 \\
\hline Neurogenic tumor & 492 & $1(0.2)$ & 0 & $1(0.2)$ & 461 \\
\hline Congenital cyst & 1224 & 0 & 0 & 0 & 1129 \\
\hline Goiter & 98 & 0 & 0 & $1(1.0)$ & 40 \\
\hline Lymphatic tumor & 172 & 0 & 0 & $1(0.6)$ & 122 \\
\hline Excision of pleural recurrence of thymoma & 20 & 0 & 0 & 0 & 15 \\
\hline Thymolipoma & 20 & 0 & 0 & 0 & 17 \\
\hline Others & 788 & 0 & $1(0.1)$ & 0 & 609 \\
\hline
\end{tabular}

(), mortality $\%$

Table 18 Thymectomy for myasthenia gravis

Table 19 Operations for nonneoplastic diseases

\begin{tabular}{llllll}
\hline & Cases & \multicolumn{2}{l}{30 -Day mortality } & Hospital mortality & By VATS \\
\cline { 3 - 5 } & & Hospital & After discharge & \\
\hline Thymectomy for myasthenia gravis & 499 & 0 & 0 & 0 & 319 \\
With thymoma & 348 & 0 & 0 & 0 & 209 \\
\hline
\end{tabular}

(), mortality \%

\begin{tabular}{|c|c|c|c|c|c|c|c|}
\hline \multirow[b]{3}{*}{ Operations for non-neoplastic diseases } & \multirow[t]{2}{*}{ Cases } & \multicolumn{4}{|c|}{ 30-Day mortality } & \multirow{2}{*}{\multicolumn{2}{|c|}{ Hospital mortality }} \\
\hline & & \multicolumn{2}{|c|}{ Hospital } & \multicolumn{2}{|c|}{ After discharge } & & \\
\hline & 22,996 & 229 & $(1.0)$ & 30 & $(0.1)$ & 465 & (2.0) \\
\hline & \multirow[t]{2}{*}{ Cases } & \multicolumn{3}{|c|}{ 30-Day mortality } & \multirow{2}{*}{\multicolumn{2}{|c|}{ Hospital mortality }} & \multirow[t]{2}{*}{ VATS } \\
\hline & & Hospital & \multicolumn{2}{|c|}{ After discharge } & & & \\
\hline A. Inflammatory pulmonary disease & 2400 & $8(0.3)$ & \multicolumn{2}{|c|}{$4(0.2)$} & \multicolumn{2}{|c|}{$18(0.8)$} & 2102 \\
\hline Tuberculous infection & 54 & $1(1.9)$ & \multicolumn{2}{|c|}{0} & \multicolumn{2}{|c|}{$1(1.9)$} & 46 \\
\hline Mycobacterial infection & 526 & $2(0.4)$ & \multicolumn{2}{|l|}{0} & \multicolumn{2}{|c|}{$3(0.6)$} & 465 \\
\hline Fungal infection & 325 & $1(0.3)$ & \multicolumn{2}{|c|}{$2(0.6)$} & \multicolumn{2}{|c|}{$6(1.8)$} & 241 \\
\hline Bronchiectasis & 64 & 0 & \multicolumn{2}{|c|}{0} & \multicolumn{2}{|l|}{0} & 48 \\
\hline Tuberculous nodule & 70 & 0 & \multicolumn{2}{|l|}{0} & \multicolumn{2}{|l|}{0} & 65 \\
\hline Inflammatory pseudotumor & 902 & 0 & \multicolumn{2}{|l|}{0} & \multicolumn{2}{|c|}{$3(0.3)$} & 838 \\
\hline Interpulmonary lymph node & 59 & 0 & \multicolumn{2}{|l|}{0} & \multicolumn{2}{|l|}{0} & 58 \\
\hline Others & 400 & $4(1.0)$ & $2(0.5$ & & $5(1$ & & 341 \\
\hline
\end{tabular}

(), mortality \% 
Table 20 B. Empyema

\begin{tabular}{clllll}
\hline & Cases & \multicolumn{2}{l}{ 30-day mortality } & Hospital mortality & by VATS \\
\cline { 3 - 4 } & & \multicolumn{2}{l}{ hospital } & After discharge & \\
\hline Acute empyema & 2402 & $57(2.4)$ & $4(0.2)$ & $124(5.2)$ & 2013 \\
With fistula & 509 & $34(6.7)$ & $1(0.2)$ & $66(13.0)$ & 270 \\
Without fistula & 1876 & $22(1.2)$ & $3(0.2)$ & $54(2.9)$ & 1729 \\
Unknown & 17 & $1(5.9)$ & 0 & $4(23.5)$ & 14 \\
Chronic empyema & 701 & $23(3.3)$ & $1(0.1)$ & $63(9.0)$ & 407 \\
With fistula & 325 & $14(4.3)$ & 0 & $36(11.1)$ & 125 \\
Without fistula & 324 & $8(2.5)$ & $1(0.3)$ & $25(7.7)$ & 241 \\
Unknown & 52 & $1(1.9)$ & 0 & $2(3.8)$ & 41 \\
Total & 3103 & $80(2.6)$ & $5(0.2)$ & $187(6.0)$ & 2420 \\
\hline
\end{tabular}

(), mortality \%

Table 21 C. Descending necrotizing mediastinitis

\begin{tabular}{llllll}
\hline & Cases & \multicolumn{3}{l}{30 -day mortality } & Hospital mortality \\
\cline { 5 - 6 } & & Hospital & After discharge & VATS \\
\hline C. Descending necrotizing mediastinitis & 106 & $4(3.8)$ & $1(0.9)$ & $6(5.7)$ & 81 \\
\hline
\end{tabular}

(), mortality $\%$

Table 22 D. Bullous diseases

\begin{tabular}{llllll}
\hline & Cases & \multicolumn{2}{l}{30 -Day mortality } & Hospital mortality & \multirow{2}{*}{ VATS } \\
\cline { 3 - 4 } & & Hospital & After discharge & \\
\hline D. Bullous diseases & 376 & 0 & 0 & 0 & 349 \\
Emphysematous bulla & 270 & 0 & 0 & 0 & 258 \\
Bronchogenic cyst & 21 & 0 & 0 & 0 & 18 \\
Emphysema with LVRS & 23 & 0 & 0 & 0 & 19 \\
Others & 62 & 0 & 0 & 0 & 54 \\
\hline
\end{tabular}

(), mortality \%

LVRS lung volume reduction surgery 
Table 23 E. Pneumothorax

\begin{tabular}{|c|c|c|c|c|c|c|}
\hline \multirow[t]{2}{*}{ Cases } & \multicolumn{3}{|l|}{ 30-day mortality } & \multicolumn{2}{|c|}{ Hospital mortality } & \multirow[t]{2}{*}{ VATS } \\
\hline & Hospital & \multicolumn{2}{|c|}{ After discharge } & & & \\
\hline 14,731 & $81(0.5)$ & \multicolumn{2}{|c|}{$19(0.1)$} & \multicolumn{2}{|c|}{$146(1.0)$} & 14,379 \\
\hline \multicolumn{7}{|c|}{ Spontaneous pneumothorax } \\
\hline \multirow{2}{*}{\multicolumn{2}{|c|}{ Operative procedure }} & \multirow[t]{2}{*}{ Cases } & \multicolumn{2}{|c|}{ 30-Day mortality } & \multirow[t]{2}{*}{ Hospital mortality } & \multirow[t]{2}{*}{ VATS } \\
\hline & & & Hospital & After discharge & & \\
\hline \multicolumn{2}{|c|}{ Bullectomy } & 2825 & $7(0.2)$ & $4(0.1)$ & $11(0.4)$ & 2,770 \\
\hline \multicolumn{2}{|c|}{ Bullectomy with additional procedure } & 7632 & $5(0.1)$ & $1(0.01)$ & $10(0.1)$ & 7,535 \\
\hline \multicolumn{2}{|c|}{ Coverage with artificial material } & 7383 & $5(0.1)$ & $1(0.01)$ & $10(0.1)$ & 7,291 \\
\hline \multicolumn{2}{|c|}{ Parietal pleurectomy } & 27 & 0 & 0 & 0 & 27 \\
\hline \multicolumn{2}{|c|}{ Coverage and parietal pleurectomy } & 57 & 0 & 0 & 0 & 54 \\
\hline \multicolumn{2}{|l|}{ Others } & 165 & 0 & 0 & 0 & 163 \\
\hline Others & & 657 & $3(0.5)$ & $2(0.3)$ & $5(0.8)$ & 610 \\
\hline Unknow & & 10 & 0 & 0 & 0 & 9 \\
\hline Total & & 11,124 & $15(0.1)$ & $7(0.1)$ & $26(0.2)$ & 10,924 \\
\hline Seconda & & & & & & \\
\hline Associat & & Cases & 30-Day 1 & lity & Hospital mortality & VATS \\
\hline & & & Hospital & After discharge & & \\
\hline COPD & & 2,437 & $33(1.4)$ & $8(0.3)$ & $65(2.7)$ & 2,359 \\
\hline Tumorou & & 128 & $9(7.0)$ & $1(0.8)$ & $14(10.9)$ & 123 \\
\hline Catamen & & 199 & 0 & 0 & $1(0.5)$ & 194 \\
\hline LAM & & 39 & 0 & 0 & 0 & 38 \\
\hline Others $(\mathrm{e}$ & horax by trauma) & 804 & $24(3.0)$ & $3(0.4)$ & $40(5.0)$ & 741 \\
\hline Unknowı & & 0 & 0 & 0 & 0 & 0 \\
\hline Operativ & & Cases & 30 Day mort & & Hospital mortality & VATS \\
\hline & & & Hospital & After discharge & & \\
\hline Bullector & & 607 & $5(0.8)$ & $1(0.2)$ & $8(1.3)$ & 587 \\
\hline Bullector & procedure & 2,079 & 33 (1.6) & $5(0.2)$ & $50(2.4)$ & 2,030 \\
\hline Covera & naterial & 1,969 & $32(1.6)$ & $5(0.3)$ & $49(2.5)$ & 1,924 \\
\hline Parietal & & 4 & 0 & 0 & 0 & 4 \\
\hline Covera & urectomy & 37 & 0 & 0 & 0 & 35 \\
\hline Others & & 69 & $1(1.4)$ & 0 & $1(1.4)$ & 67 \\
\hline Others & & 917 & $28(3.1)$ & $6(0.7)$ & $62(6.8)$ & 836 \\
\hline Unknowr & & 4 & 0 & 0 & 0 & 2 \\
\hline Total & & 3607 & $66(1.8)$ & $12(0.3)$ & $120(3.3)$ & 3,455 \\
\hline
\end{tabular}

(), mortality $\%$ 
Table 24 F. Chest wall deformity

\begin{tabular}{lllll}
\hline & Cases & \multicolumn{2}{l}{30 -Day mortality } & $\begin{array}{l}\text { Hospital } \\
\text { mortality }\end{array}$ \\
\cline { 3 - 4 } & & Hospital $\begin{array}{l}\text { After } \\
\text { discharge }\end{array}$ & \\
\hline $\begin{array}{l}\text { F. Chest wall } \\
\text { deformity }\end{array}$ & 176 & 0 & 0 & $1(0.6)$ \\
$\begin{array}{l}\text { Funnel chest } \\
\text { Others }\end{array}$ & 165 & 0 & 0 & $1(0.6)$ \\
\hline
\end{tabular}

(), mortality $\%$

Table 25 G. Diaphragmatic hernia

\begin{tabular}{llllll}
\hline & Cases & \multicolumn{2}{l}{ 30-Day mortality } & $\begin{array}{l}\text { Hospital } \\
\text { mortality }\end{array}$ & VATS \\
\cline { 2 - 5 } & & Hospital & $\begin{array}{l}\text { After } \\
\text { discharge }\end{array}$ \\
\hline $\begin{array}{l}\text { G. } \\
\quad \begin{array}{l}\text { Diaphragmatic } \\
\text { hernia }\end{array}\end{array}$ & 30 & $1(3.3)$ & 0 & $3(10.0)$ & 21 \\
Congenital & 5 & 0 & 0 & $2(40.0)$ & 4 \\
Traumatic & 4 & 0 & 0 & 0 & 3 \\
Others & 21 & $1(4.8)$ & 0 & $1(4.8)$ & 14 \\
\hline
\end{tabular}

(), mortality $\%$

Table $26 \mathrm{H}$. Chest trauma

\begin{tabular}{llllll}
\hline & Cases & \multicolumn{2}{l}{30 -Day mortality } & $\begin{array}{l}\text { Hospital } \\
\text { mortality }\end{array}$ & VATS \\
\cline { 2 - 5 } & & Hospital & $\begin{array}{l}\text { After } \\
\text { discharge }\end{array}$ & \\
\hline $\begin{array}{c}\text { H. Chest } \\
\text { trauma }\end{array}$ & 431 & $21(4.9)$ & 0 & $33(7.7)$ & 280 \\
\hline
\end{tabular}

(), mortality \%
Table 28 Lung transplantation

\begin{tabular}{|c|c|c|c|c|c|}
\hline & & \multirow[t]{2}{*}{ Cases } & \multicolumn{2}{|c|}{ 30-Day mortality } & \multirow{2}{*}{$\begin{array}{l}\text { Hospital } \\
\text { mortality }\end{array}$} \\
\hline & & & Hospital & $\begin{array}{l}\text { After } \\
\text { discharge }\end{array}$ & \\
\hline \multicolumn{2}{|c|}{$\begin{array}{l}\text { Single lung transplantation } \\
\text { from brain-dead donor }\end{array}$} & 30 & 0 & 0 & 0 \\
\hline \multicolumn{2}{|c|}{$\begin{array}{l}\text { Bilateral lung } \\
\text { transplantation from } \\
\text { brain-dead donor }\end{array}$} & 27 & 0 & 0 & 0 \\
\hline \multicolumn{2}{|c|}{$\begin{array}{l}\text { Lung transplantation from } \\
\text { living donor }\end{array}$} & 14 & 0 & 0 & $1(7.1)$ \\
\hline \multicolumn{2}{|c|}{ Total lung transplantation } & 71 & 0 & 0 & $1(1.4)$ \\
\hline \multicolumn{2}{|c|}{$\begin{array}{l}\text { Donor of living donor lung } \\
\text { transplantation }\end{array}$} & 23 & 0 & 0 & 0 \\
\hline \multicolumn{6}{|l|}{ (), mortality $\%$} \\
\hline \multicolumn{6}{|c|}{ Table 29 Video-assisted thoracic surgery } \\
\hline & \multirow[t]{2}{*}{ Cases } & \multicolumn{3}{|c|}{ 30-Day mortality } & \multirow{2}{*}{$\begin{array}{l}\text { Hospital } \\
\text { mortality }\end{array}$} \\
\hline & & & pital & $\begin{array}{l}\text { After } \\
\text { discharge }\end{array}$ & \\
\hline $\begin{array}{l}\text { Video-assisted } \\
\text { thoracic surgery }\end{array}$ & 71,171 & & $(0.3)$ & $48(0.1)$ & $474(0.7)$ \\
\hline
\end{tabular}


Table 30 Tracheobronchoplasty

\begin{tabular}{|c|c|c|c|c|}
\hline & \multirow[t]{2}{*}{ Cases } & \multicolumn{2}{|c|}{ 30-Day mortality } & \multirow{2}{*}{$\begin{array}{l}\text { Hospital } \\
\text { mortality }\end{array}$} \\
\hline & & Hospital & $\begin{array}{l}\text { After } \\
\text { discharge }\end{array}$ & \\
\hline Tracheobronchoplasty & 747 & $8(1.1)$ & $1(0.1)$ & $11(1.5)$ \\
\hline Trachea & 46 & $1(2.2)$ & 0 & $1(2.2)$ \\
\hline $\begin{array}{l}\text { Sleeve resection with } \\
\text { reconstruction }\end{array}$ & 32 & 0 & 0 & 0 \\
\hline Wedge with simple closure & 4 & 0 & 0 & 0 \\
\hline Wedge with patch closure & 1 & 0 & 0 & 0 \\
\hline $\begin{array}{l}\text { Total laryngectomy with } \\
\text { tracheostomy }\end{array}$ & 0 & 0 & 0 & 0 \\
\hline Others & 9 & $1(11.1)$ & 0 & $1(11.1)$ \\
\hline Carinal reconstruction & 35 & 0 & 0 & $1(2.9)$ \\
\hline Sleeve pneumonectomy & 10 & 0 & 0 & 0 \\
\hline Sleeve lobectomy & 464 & $1(0.2)$ & 0 & $2(0.4)$ \\
\hline Sleeve segmental excision & 15 & 0 & 0 & 0 \\
\hline $\begin{array}{l}\text { Bronchoplasty without lung } \\
\text { resection }\end{array}$ & 23 & $1(4.3)$ & $1(4.3)$ & $1(4.3)$ \\
\hline Others & 154 & $5(3.2)$ & 0 & $6(3.9)$ \\
\hline
\end{tabular}

(), mortality $\%$

Table 31 Pediatric surgery

\begin{tabular}{lllll}
\hline & Cases & \multicolumn{2}{l}{ 30-Day mortality } & $\begin{array}{l}\text { Hospital } \\
\text { mortality }\end{array}$ \\
\cline { 3 - 4 } & Hospital & $\begin{array}{l}\text { After } \\
\text { discharge }\end{array}$ & \\
\hline $\begin{array}{c}\text { Pediatric } \\
\text { surgery }\end{array}$ & 287 & $7(2.4)$ & $1(0.3)$ & $11(3.8)$ \\
\hline
\end{tabular}

(), mortality $\%$
Table 32 Combined resection of neighboring organ(s)

\begin{tabular}{|c|c|c|c|c|c|c|c|}
\hline \multirow[b]{3}{*}{$\begin{array}{r}\text { Combined resection of } \\
\text { neighboring organ }(\mathrm{s})\end{array}$} & \multirow{2}{*}{\multicolumn{2}{|c|}{ Cases }} & \multicolumn{4}{|c|}{ 30-Day mortality } & \multirow{3}{*}{$\begin{array}{l}\begin{array}{l}\text { Hospital } \\
\text { mortality }\end{array} \\
21 \quad(1.5)\end{array}$} \\
\hline & & & Hosp & jital & $\begin{array}{l}\text { Afte } \\
\text { disc }\end{array}$ & $\begin{array}{l}\text { er } \\
\text { charge }\end{array}$ & \\
\hline & \multicolumn{2}{|c|}{1401} & 10 & $(0.7)$ & 1 & $(0.1)$ & \\
\hline \multirow[t]{2}{*}{ Organ resected } & \multirow[t]{2}{*}{ Cases } & \multicolumn{5}{|c|}{ 30-Day mortality } & \multirow{2}{*}{$\begin{array}{l}\text { Hospital } \\
\text { mortality }\end{array}$} \\
\hline & & & spital & $\begin{array}{l}\text { Aft } \\
\text { disc }\end{array}$ & harge & & \\
\hline \multicolumn{8}{|l|}{ A. Primary lung cancer } \\
\hline Aorta & 10 & \multicolumn{2}{|c|}{$1(10.0)$} & 0 & & & $1(10.0)$ \\
\hline Superior vena cava & 21 & \multicolumn{2}{|l|}{0} & 0 & & & $1(4.8)$ \\
\hline $\begin{array}{l}\text { Brachiocephalic } \\
\text { vein }\end{array}$ & 8 & \multicolumn{2}{|l|}{0} & 0 & & & $1(12.5)$ \\
\hline Pericardium & 122 & \multicolumn{2}{|c|}{$1(0.8)$} & 0 & & & $4(3.3)$ \\
\hline Pulmonary artery & 146 & \multicolumn{2}{|c|}{$1(0.7)$} & 0 & & & $2(1.4)$ \\
\hline Left atrium & 18 & \multicolumn{2}{|l|}{0} & 0 & & & 0 \\
\hline Diaphragm & 74 & \multicolumn{2}{|c|}{$1(1.4)$} & 0 & & & $1(1.4)$ \\
\hline $\begin{array}{l}\text { Chest wall } \\
\text { (including ribs) }\end{array}$ & 330 & \multicolumn{2}{|c|}{$5(1.5)$} & 0 & & & $9(2.7)$ \\
\hline Vertebra & 8 & \multicolumn{2}{|l|}{0} & 0 & & & 0 \\
\hline Esophagus & 4 & \multicolumn{2}{|l|}{0} & 0 & & & 0 \\
\hline Total & 741 & & $(1.2)$ & 0 & & & $19(2.6)$ \\
\hline B. Mediastinal tumor & & & & & & & \\
\hline Aorta & 6 & & $(16.7)$ & 0 & & & $1(16.7)$ \\
\hline Superior vena cava & 53 & 0 & & 0 & & & $1(1.9)$ \\
\hline $\begin{array}{l}\text { Brachiocephalic } \\
\text { vein }\end{array}$ & 112 & 0 & & 0 & & & $1(0.9)$ \\
\hline Pericardium & 336 & 0 & & $1(C$ & .3) & & $1(0.3)$ \\
\hline Pulmonary artery & 4 & 1( & $(25.0)$ & 0 & & & $1(25.0)$ \\
\hline Left atrium & 2 & 0 & & 0 & & 0 & 0 \\
\hline Diaphragm & 30 & 0 & & 0 & & 0 & 0 \\
\hline $\begin{array}{l}\text { Chest wall } \\
\text { (including ribs) }\end{array}$ & 4 & 0 & & 0 & & 0 & 0 \\
\hline Vertebra & 5 & 0 & & 0 & & 0 & 0 \\
\hline Esophagus & 4 & 0 & & 0 & & 0 & 0 \\
\hline Lung & 487 & & $(0.2)$ & $1(C$ & .2) & & $2(0.4)$ \\
\hline Total & 1,043 & & $(0.3)$ & $2(0$ & .2) & & $7(0.7)$ \\
\hline
\end{tabular}

(), mortality \%

Table 33 Operation of lung cancer invading the chest wall of the apex

\begin{tabular}{lllll}
\hline & Cases & \multicolumn{2}{l}{ 30-Day mortality } & $\begin{array}{l}\text { Hospital } \\
\text { mortality }\end{array}$ \\
\cline { 2 - 4 } & & Hospital $\begin{array}{l}\text { After } \\
\text { discharge }\end{array}$ & \\
\hline $\begin{array}{l}\text { 15. Operation of lung } \\
\text { cancer invading the } \\
\text { chest wall of the apex }\end{array}$ & 772 & $6(0.8)$ & 0 & $9(1.2)$ \\
\hline
\end{tabular}

(), mortality $\%$

Includes tumors invading the anterior apical chest wall and posterior apical chest wall (superior sulcus tumor, so called Pancoast type) 
Details regarding tracheobronchoplasty, pediatric surgery, and combined resection of neighboring organs are presented in Tables 30, 31, 32, 33.

\section{(C) Esophageal surgery}

In 2018, the data collection method for esophageal surgery had been modified from self-reports using questionnaire sheets according to each institution belonging to the Japanese Association for Thoracic Surgery to an automatic package downloaded from the NCD in Japan. Consequently,

Table 34 Distribution of number of esophageal operations in 2018 in each institution

\begin{tabular}{llll}
\hline \multicolumn{2}{l}{ Esophageal surgery } & \\
\hline $\begin{array}{l}\text { Number of } \\
\text { operations in } \\
2018\end{array}$ & $\begin{array}{l}\text { Benign } \\
\text { esophageal } \\
\text { diseases }\end{array}$ & $\begin{array}{l}\text { Malignant } \\
\text { Esophageal } \\
\text { disease }\end{array}$ & Benign + Malignant \\
\hline 0 & 224 & 111 & 63 \\
$1-4$ & 271 & 179 & 193 \\
$5-9$ & 43 & 92 & 101 \\
$10-19$ & 10 & 88 & 95 \\
$20-29$ & 0 & 36 & 38 \\
$30-39$ & 1 & 10 & 21 \\
$40-49$ & 2 & 13 & 14 \\
$\geqq 50$ & 1 & 23 & 27 \\
Total & 552 & 552 & 552 \\
\hline
\end{tabular}

data for non-surgical cases with esophageal diseases had been excluded from the registry. Furthermore, data regarding the histological classification of malignant tumors, multiple primary cancers, and mortality rates for cases with combined resection of other organs could not be registered given that they were not included in the NCD. Instead, detailed data regarding postoperative surgical and non-surgical complications were collected from the NCD. Moreover, data regarding surgeries for corrosive esophageal strictures and salvage surgeries for esophageal cancer had been exceptionally registered by participating institutions.

Throughout 2018, a total of 7324 patients underwent surgery for esophageal diseases (1068 and 6256 for benign and malignant esophageal diseases, respectively) from 552 institutions across Japan. Among them, 329 (63.0\%) and $441(79.9 \%)$ institutions performed surgeries for benign and malignant esophageal diseases, respectively. Among institutions performing surgeries for malignant esophageal diseases, $82(18.6 \%)$ had 20 or more patients who underwent esophageal surgeries within 2018, while 271 (61.5\%) had less than 10 patients (i.e., 1-9 patients) who underwent the same procedure within the same year. This distribution was quite different from that in 2017 [125 (29.2\%) and 215 $(50.2 \%)$, respectively], suggesting the differences between the two data collection methods, as mentioned previously (Table 34). Annual trends among registered in-patients with esophageal diseases have remained unchanged for the past 5 years (Fig. 3).

With regard to benign esophageal diseases (Table 35), thoracoscopic and/or laparoscopic surgeries were

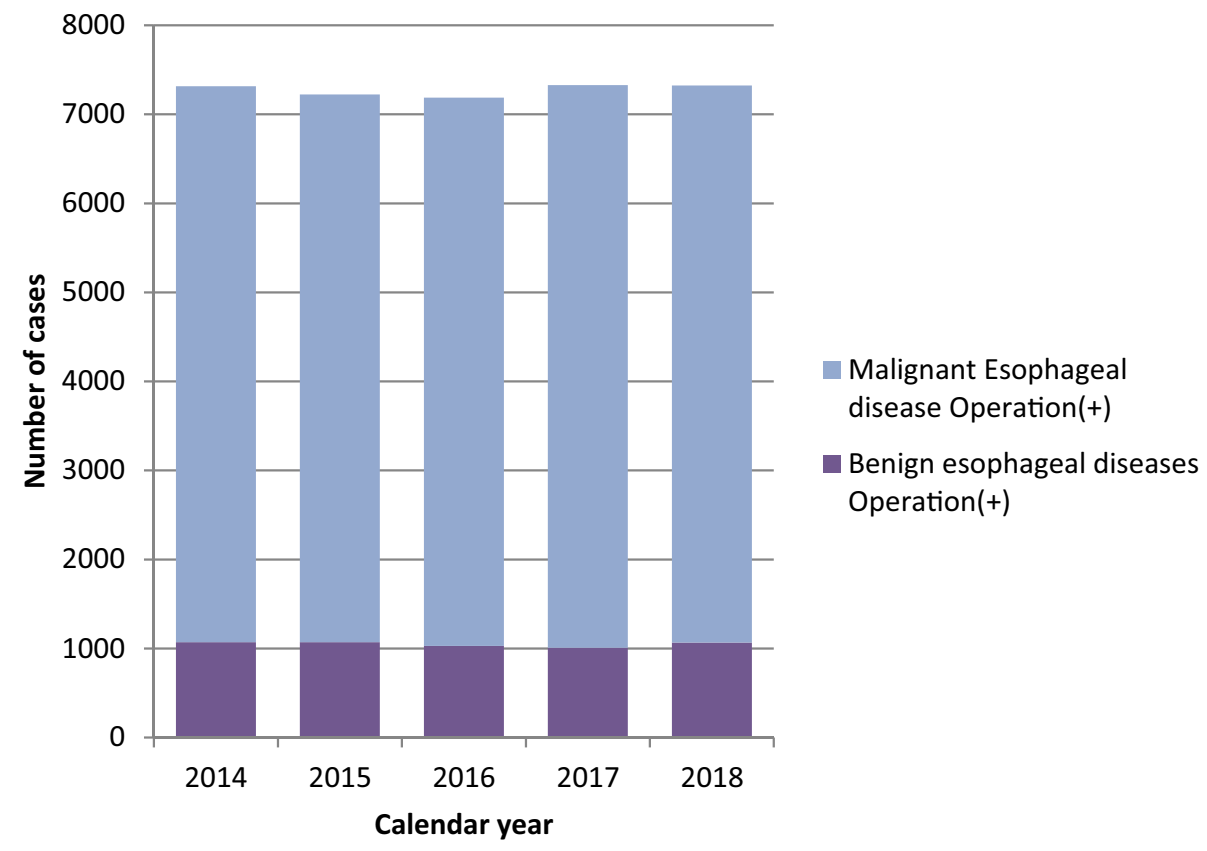

Fig. 3 Annual trend of in-patients with esophageal diseases 


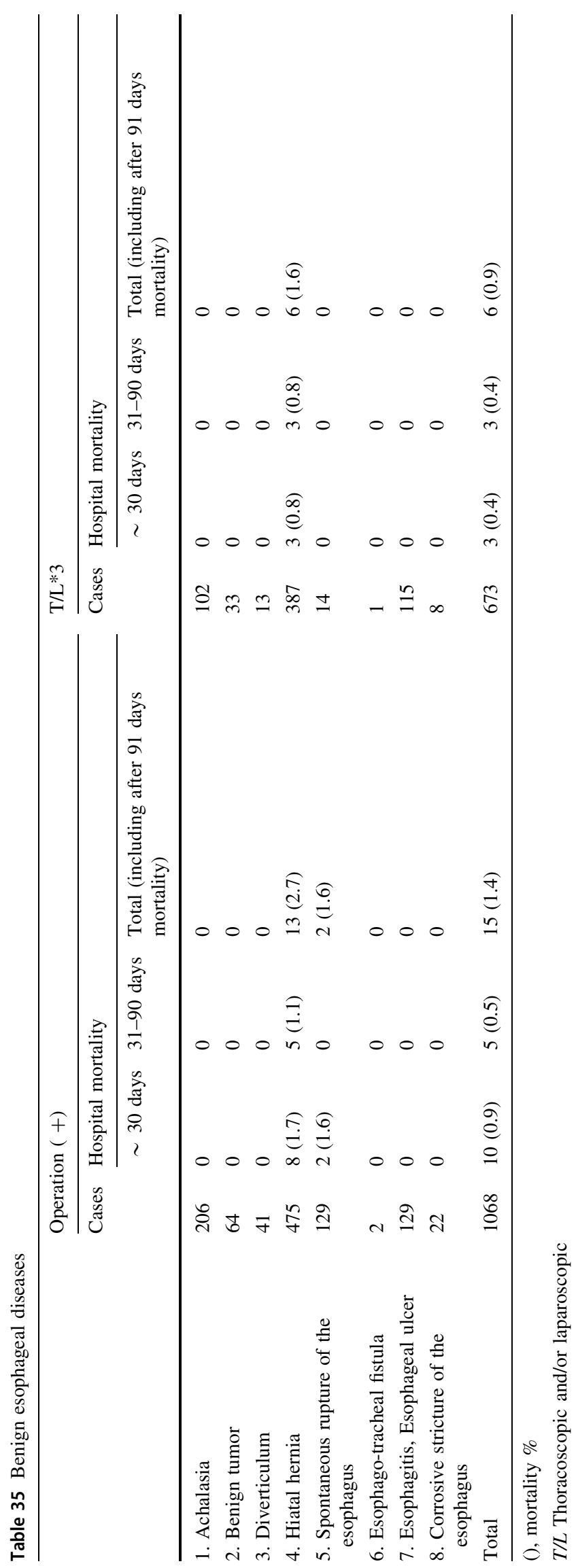


Table 36 Malignant esophageal disease

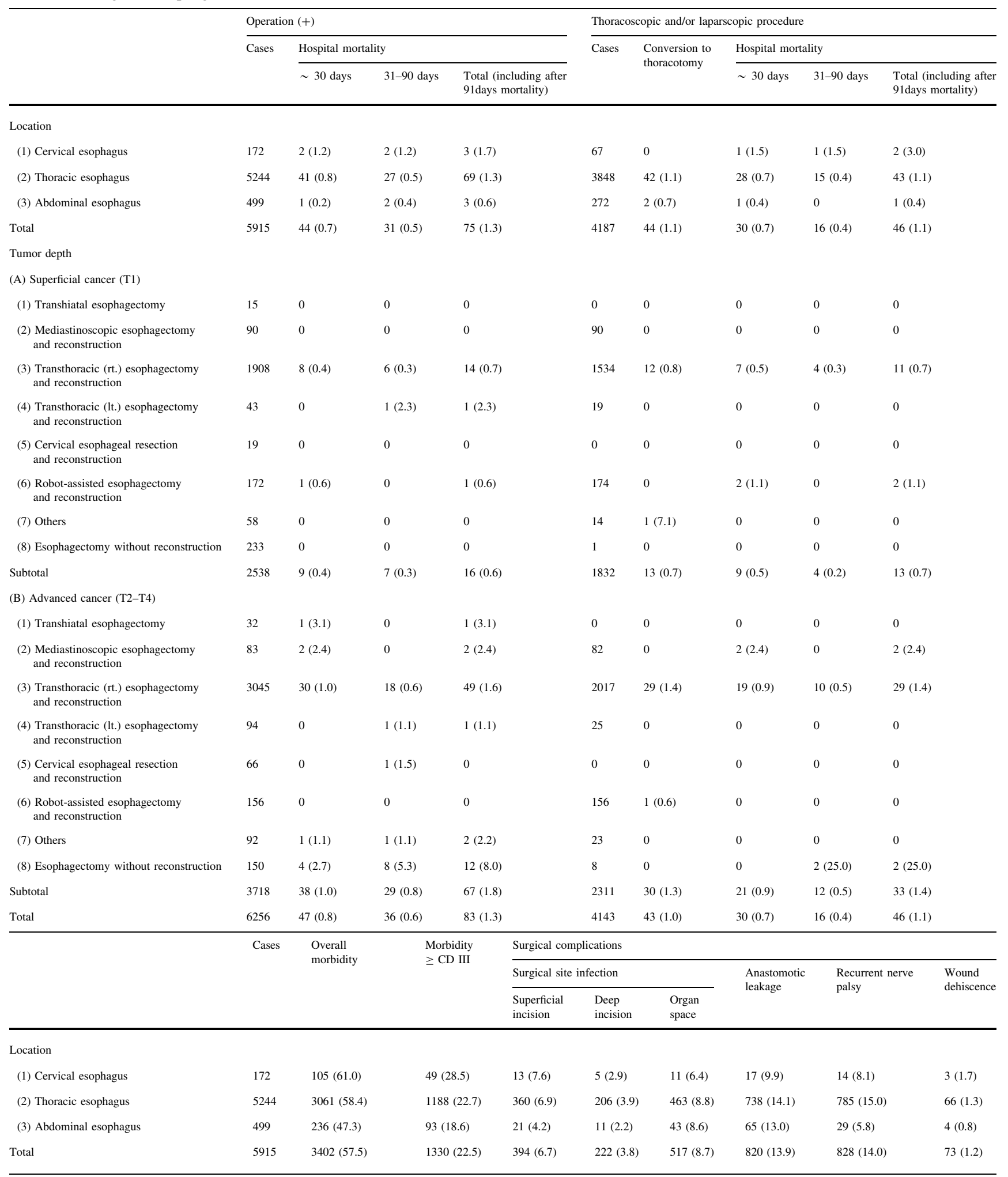


Table 36 (continued)

\begin{tabular}{|c|c|c|c|c|c|c|c|c|c|c|c|c|}
\hline & \multirow[t]{3}{*}{ Cases } & \multirow{3}{*}{\multicolumn{2}{|c|}{$\begin{array}{l}\text { Overall } \\
\text { morbidity }\end{array}$}} & \multirow{3}{*}{$\begin{array}{l}\text { Morbidity } \\
\geq \text { CD III }\end{array}$} & \multicolumn{8}{|c|}{ Surgical complications } \\
\hline & & & & & \multicolumn{4}{|c|}{ Surgical site infection } & \multirow{2}{*}{$\begin{array}{l}\text { Anastomotic } \\
\text { leakage }\end{array}$} & \multirow{2}{*}{\multicolumn{2}{|c|}{$\begin{array}{l}\text { Recurrent nerve } \\
\text { palsy }\end{array}$}} & \multirow{2}{*}{$\begin{array}{l}\text { Wound } \\
\text { dehiscence }\end{array}$} \\
\hline & & & & & $\begin{array}{l}\text { Superficial } \\
\text { incision }\end{array}$ & $\begin{array}{l}\text { Deep } \\
\text { incision }\end{array}$ & $\begin{array}{l}\text { Or } \\
\text { sp }\end{array}$ & & & & & \\
\hline \multicolumn{13}{|l|}{ Tumor depth } \\
\hline \multicolumn{13}{|l|}{ (A) Superficial cancer (T1) } \\
\hline (1) Transhiatal esophagectomy & 15 & 10 & $(66.7)$ & $7(46.7)$ & $2(13.3)$ & $2(13.3)$ & \multicolumn{2}{|c|}{$3(20.0)$} & $5(33.3)$ & \multicolumn{2}{|r|}{$1(6.7)$} & 0 \\
\hline $\begin{array}{l}\text { (2) Mediastinoscopic esophagectomy } \\
\text { and reconstruction }\end{array}$ & 90 & 54 & $(60.0)$ & $20(22.2)$ & $7(7.8)$ & $2(2.2)$ & \multicolumn{2}{|c|}{$8(8.9)$} & $18(20.0)$ & \multicolumn{2}{|r|}{$22(24.4)$} & 0 \\
\hline $\begin{array}{l}\text { (3) Transthoracic (rt.) esophagectomy } \\
\text { and reconstruction }\end{array}$ & 1908 & 1100 & $(57.7)$ & $421(22.1)$ & $129(6.8)$ & $75(3.9)$ & \multicolumn{2}{|c|}{$171(9.0)$} & $294(15.4)$ & \multicolumn{2}{|r|}{$274(14.4)$} & $29(1.5)$ \\
\hline $\begin{array}{l}\text { (4) Transthoracic (lt.) esophagectomy } \\
\text { and reconstruction }\end{array}$ & 43 & 19 & $(44.2)$ & $9(20.9)$ & $2(4.7)$ & $1(2.3)$ & 4 & & $5(11.6)$ & & $1(2.3)$ & 0 \\
\hline $\begin{array}{l}\text { (5) Cervical esophageal resection } \\
\text { and reconstruction }\end{array}$ & 19 & 15 & (78.9) & $3(15.8)$ & $1(5.3)$ & 0 & & & $2(10.5)$ & & $3(15.8)$ & 0 \\
\hline $\begin{array}{l}\text { (6) Robot-assisted esophagectomy } \\
\text { and reconstruction }\end{array}$ & 172 & $99(5$ & & $35(20.3)$ & $6(3.5)$ & $2(1.2)$ & & (7.6) & $25(14.5)$ & & $25(14.5)$ & $1(0.6)$ \\
\hline (7) Others & 58 & $30(5$ & & $9(15.5)$ & 0 & 0 & 5 & & $11(19.0)$ & 0 & 0 & 0 \\
\hline (8) Esophagectomy without reconstruction & 233 & $32(1$ & & $10(4.3)$ & 0 & 0 & 0 & & 0 & 0 & 0 & 0 \\
\hline Subtotal & 253 & 1359 & $(53.5)$ & $514(20.3)$ & $147(5.8)$ & $82(3.2)$ & & $(8.1)$ & $360(14.2)$ & & $326(12.8)$ & $30(1.2)$ \\
\hline (B) Advanced cancer (T2-T4) & & & & & & & & & & & & \\
\hline (1) Transhiatal esophagectomy & 32 & $18(5$ & & $9(28.1)$ & 0 & $4(12.5)$ & & 2.5) & $6(18.8)$ & & $2(6.3)$ & 0 \\
\hline $\begin{array}{l}\text { (2) Mediastinoscopic esophagectomy } \\
\text { and reconstruction }\end{array}$ & 83 & $55(6$ & & $21(25.3)$ & $4(4.8)$ & $2(2.4)$ & & & $16(19.3)$ & & $20(24.1)$ & 0 \\
\hline $\begin{array}{l}\text { (3) Transthoracic (rt.) esophagectomy } \\
\text { and reconstruction }\end{array}$ & 304 & 1749 & (57.4) & $696(22.9)$ & $221(7.3)$ & $121(4.0)$ & & (8.7) & $386(12.7)$ & & $422(13.9)$ & $38(1.2)$ \\
\hline $\begin{array}{l}\text { (4) Transthoracic (lt.) esophagectomy } \\
\text { and reconstruction }\end{array}$ & 94 & $51(5$ & & $23(24.5)$ & $4(4.3)$ & $6(6.4)$ & & & $10(10.6)$ & & $7(7.4)$ & 0 \\
\hline $\begin{array}{l}\text { (5) Cervical esophageal resection } \\
\text { and reconstruction }\end{array}$ & 66 & $41(6$ & & $15(22.7)$ & $9(13.6)$ & $3(4.5)$ & & & $4(6.1)$ & & $7(10.6)$ & 0 \\
\hline $\begin{array}{l}\text { (6) Robot-assisted esophagectomy } \\
\text { and reconstruction }\end{array}$ & 156 & $87(5$ & & $25(16.0)$ & $6(3.8)$ & $2(1.3)$ & & & $17(10.9)$ & & $36(23.1)$ & 0 \\
\hline (7) Others & 92 & $45(4$ & & $19(20.7)$ & 0 & 0 & & 12.0) & $13(14.1)$ & & $4(4.3)$ & $2(2.2)$ \\
\hline (8) Esophagectomy without reconstruction & 150 & $77(5$ & & $38(25.3)$ & $1(0.7)$ & 0 & & & 0 & 0 & 0 & $1(0.7)$ \\
\hline Subtotal & 371 & 2123 & $(57.1)$ & $846(22.8)$ & $245(6.6)$ & $138(3.7)$ & & (8.2) & $452(12.2)$ & & 498 (13.4) & $41(1.1)$ \\
\hline Total & 625 & 3482 & (55.7) & $1360(21.7)$ & $392(6.3)$ & $220(3.5)$ & & (8.2) & $812(13.0)$ & & $824(13.2)$ & $71(1.1)$ \\
\hline & Cases & Nonsurgical & complications & & & & & & & & Readmission & Reoperation \\
\hline & & Pneumonia & $\begin{array}{l}\text { Unplanned } \\
\text { intubation }\end{array}$ & $\begin{array}{l}\text { prolonged } \\
\text { ventilation }>48 \mathrm{~h}\end{array}$ & $\begin{array}{l}\text { pulmonary } \\
\text { embolism }\end{array}$ & atelectasis & $\begin{array}{l}\text { Renal } \\
\text { failure }\end{array}$ & $\begin{array}{l}\text { CNS } \\
\text { events }\end{array}$ & $\begin{array}{l}\text { Cardiac } \\
\text { events }\end{array}$ & $\begin{array}{l}\text { Septic } \\
\text { shock }\end{array}$ & 30 days & 30 days \\
\hline Location & & & & & & & & & & & & \\
\hline (1) Cervical esophagus & 172 & $16(9.3)$ & $12(7.0)$ & $18(10.5)$ & $5(2.9)$ & $7(4.1)$ & $3(1.7)$ & $1(0.6)$ & $3(1.7)$ & $3(1.7)$ & $3(1.7)$ & $17(9.9)$ \\
\hline (2) Thoracic esophagus & 5244 & $798(15.2)$ & $269(5.1)$ & $337(6.4)$ & $38(0.7)$ & $302(5.8)$ & $27(0.5)$ & $27(0.5)$ & $20(0.4)$ & $38(0.7)$ & $134(2.6)$ & $333(6.4)$ \\
\hline (3) Abdominal esophagus & 499 & $56(11.2)$ & $17(3.4)$ & $22(4.4)$ & $2(0.4)$ & $33(6.6)$ & $3(0.6)$ & $2(0.4)$ & $1(0.2)$ & $6(1.2)$ & $16(3.2)$ & $29(5.8)$ \\
\hline Total & 5915 & $870(14.7)$ & $298(5.0)$ & $377(6.4)$ & $45(0.8)$ & $342(5.8)$ & $33(0.6)$ & $30(0.5)$ & $24(0.4)$ & $47(0.8)$ & $153(2.6)$ & $379(6.4)$ \\
\hline Tumor depth & & & & & & & & & & & & \\
\hline (A) Superficial cancer (T1) & & & & & & & & & & & & \\
\hline (1) Transhiatal esophagectomy & 15 & $5(33.3)$ & 0 & 0 & 0 & $2(13.3)$ & 0 & 0 & 0 & $1(6.7)$ & $1(6.7)$ & $3(20.0)$ \\
\hline $\begin{array}{l}\text { (2) Mediastinoscopic esophagectomy } \\
\text { and reconstruction }\end{array}$ & 90 & $10(11.1)$ & $6(6.7)$ & $8(8.9)$ & 0 & $3(3.3)$ & $1(1.1)$ & 0 & $1(1.1)$ & $1(1.1)$ & $2(2.2)$ & $5(5.6)$ \\
\hline $\begin{array}{l}\text { (3) Transthoracic (rt.) esophagectomy } \\
\text { and reconstruction }\end{array}$ & 1908 & $281(14.7)$ & $84(4.4)$ & $105(5.5)$ & $20(1.0)$ & $97(5.1)$ & $10(0.5)$ & $9(0.5)$ & $6(0.3)$ & $13(0.7)$ & $44(2.3)$ & $123(6.4)$ \\
\hline $\begin{array}{l}\text { (4) Transthoracic (lt.) esophagectomy } \\
\text { and reconstruction }\end{array}$ & 43 & $4(9.3)$ & $2(4.7)$ & $3(7.0)$ & 0 & $3(7.0)$ & 0 & 0 & 0 & 0 & $1(2.3)$ & $2(4.7)$ \\
\hline $\begin{array}{l}\text { (5) Cervical esophageal resection } \\
\text { and reconstruction }\end{array}$ & 19 & $3(15.8)$ & $1(5.3)$ & $1(5.3)$ & 0 & $2(10.5)$ & 0 & $1(5.3)$ & 0 & 0 & 0 & $1(5.3)$ \\
\hline $\begin{array}{l}\text { (6) Robot-assisted esophagectomy } \\
\text { and reconstruction }\end{array}$ & 172 & $21(12.2)$ & $5(2.9)$ & $9(5.2)$ & $3(1.7)$ & $3(1.7)$ & $3(1.7)$ & $2(1.2)$ & 0 & 0 & $3(1.7)$ & $3(1.7)$ \\
\hline
\end{tabular}


Table 36 (continued)

\begin{tabular}{|c|c|c|c|c|c|c|c|c|c|c|c|c|}
\hline & \multirow[t]{2}{*}{ Cases } & \multicolumn{9}{|c|}{ Nonsurgical complications } & \multirow{2}{*}{$\begin{array}{l}\text { Readmission } \\
\text { within } \\
30 \text { days }\end{array}$} & \multirow{2}{*}{$\begin{array}{l}\text { Reoperation } \\
\text { within } \\
30 \text { days }\end{array}$} \\
\hline & & Pneumonia & $\begin{array}{l}\text { Unplanned } \\
\text { intubation }\end{array}$ & $\begin{array}{l}\text { prolonged } \\
\text { ventilation }>48 \mathrm{~h}\end{array}$ & $\begin{array}{l}\text { pulmonary } \\
\text { embolism }\end{array}$ & atelectasis & $\begin{array}{l}\text { Renal } \\
\text { failure }\end{array}$ & $\begin{array}{l}\text { CNS } \\
\text { events }\end{array}$ & $\begin{array}{l}\text { Cardiac } \\
\text { events }\end{array}$ & $\begin{array}{l}\text { Septic } \\
\text { shock }\end{array}$ & & \\
\hline (7) Others & 58 & $7(12.1)$ & $1(1.7)$ & $1(1.7)$ & $1(1.7)$ & $5(8.6)$ & 0 & 0 & 0 & 0 & $2(3.4)$ & $4(6.9)$ \\
\hline $\begin{array}{l}\text { (8) Esophagectomy without } \\
\text { reconstruction }\end{array}$ & 233 & 0 & 0 & 0 & 0 & 0 & 0 & 0 & 0 & 0 & $4(1.7)$ & 0 \\
\hline Subtotal & 2538 & $331(13.0)$ & $99(3.9)$ & $127(5.0)$ & $24(0.9)$ & $115(4.5)$ & $14(0.6)$ & $12(0.5)$ & $7(0.3)$ & $15(0.6)$ & $57(2.2)$ & $141(5.6)$ \\
\hline \multicolumn{13}{|l|}{ (B) Advanced cancer (T2-T4) } \\
\hline (1) Transhiatal esophagectomy & 32 & $1(3.1)$ & $1(3.1)$ & $3(9.4)$ & 0 & $1(3.1)$ & 0 & 0 & $1(3.1)$ & 0 & 0 & $3(9.4)$ \\
\hline $\begin{array}{l}\text { (2) Mediastinoscopic esophagectomy } \\
\text { and reconstruction }\end{array}$ & 83 & $7(8.4)$ & $3(3.6)$ & $6(7.2)$ & $1(1.2)$ & $2(2.4)$ & $1(1.2)$ & 0 & $1(1.2)$ & $1(1.2)$ & $1(1.2)$ & $5(6.0)$ \\
\hline $\begin{array}{l}\text { (3) Transthoracic (rt.) esophagectomy } \\
\text { and reconstruction }\end{array}$ & 3045 & $474(15.6)$ & $177(5.8)$ & $213(7.0)$ & $15(0.5)$ & $200(6.6)$ & $15(0.5)$ & $14(0.5)$ & $13(0.4)$ & $26(0.9)$ & $89(2.9)$ & $202(6.6)$ \\
\hline $\begin{array}{l}\text { (4) Transthoracic (lt.) esophagectomy } \\
\text { and reconstruction }\end{array}$ & 94 & $15(16.0)$ & $4(4.3)$ & $6(6.4)$ & $2(2.1)$ & $6(6.4)$ & $1(1.1)$ & $2(2.1)$ & 0 & 0 & $5(5.3)$ & $6(6.4)$ \\
\hline $\begin{array}{l}\text { (5) Cervical esophageal resection } \\
\text { and reconstruction }\end{array}$ & 66 & $6(9.1)$ & $4(6.1)$ & $3(4.5)$ & $1(1.5)$ & 0 & 0 & $1(1.5)$ & $1(1.5)$ & 0 & 0 & $5(7.6)$ \\
\hline $\begin{array}{l}\text { (6) Robot-assisted esophagectomy } \\
\text { and reconstruction }\end{array}$ & 156 & $19(12.2)$ & $5(3.2)$ & $7(4.5)$ & $2(1.3)$ & $7(4.5)$ & 0 & 0 & $1(0.6)$ & 0 & $3(1.9)$ & $6(3.8)$ \\
\hline (7) Others & 92 & $8(8.7)$ & $3(3.3)$ & $6(6.5)$ & 0 & $9(9.8)$ & 0 & 0 & 0 & $1(1.1)$ & $3(3.3)$ & $6(6.5)$ \\
\hline $\begin{array}{l}\text { (8) Esophagectomy without } \\
\text { reconstruction }\end{array}$ & 150 & $3(2.0)$ & $2(1.3)$ & $3(2.0)$ & 0 & $1(0.7)$ & 0 & $1(0.7)$ & 0 & $2(1.3)$ & $6(4.0)$ & $3(2.0)$ \\
\hline Subtotal & 3718 & $533(14.3)$ & $199(5.4)$ & 247 (6.6) & $21(0.6)$ & $226(6.1)$ & $17(0.5)$ & $18(0.5)$ & $17(0.5)$ & $30(0.8)$ & 107 (2.9) & $236(6.3)$ \\
\hline Total & 6256 & $864(13.8)$ & $298(4.8)$ & $374(6.0)$ & $45(0.7)$ & $341(5.5)$ & $31(0.5)$ & $30(0.5)$ & $24(0.4)$ & $45(0.7)$ & $164(2.6)$ & $377(6.0)$ \\
\hline
\end{tabular}

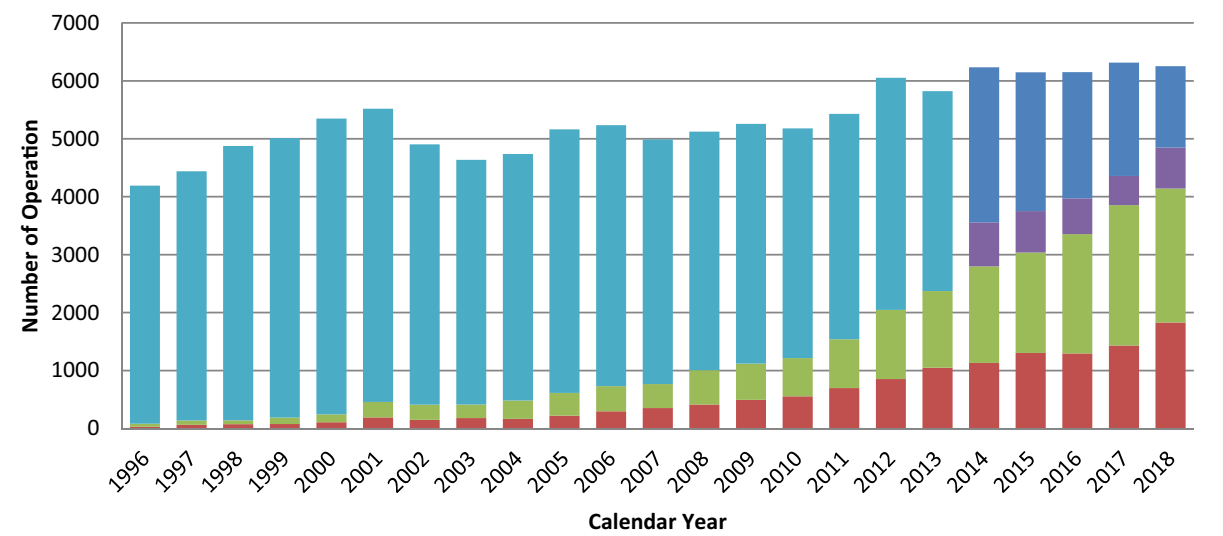

- Open

- Open for advanced esophageal cancer

- Open for superficical esophageal cancer

MIE for advanced esophageal cancer

- MIE for superficical esophageal cancer

Open esophagectomy fo superficial and advanced esophageal cancers has begun to be separately counted since 2014 .

$\mathrm{MIE}=$ minimally invasive esophagectomy

Fig. 4 Annual trend of esophagectomy

Table 37 Salvage surgery

\begin{tabular}{|c|c|c|c|c|c|c|c|c|c|c|}
\hline & \multicolumn{4}{|c|}{ Operation $(+)$} & \multicolumn{5}{|c|}{ Thoracoscopic and/or laparscopic procedure } & \multirow{3}{*}{$\begin{array}{l}\text { EMR } \\
\text { or } \\
\text { ESD }\end{array}$} \\
\hline & \multirow[t]{2}{*}{ Cases } & \multicolumn{3}{|c|}{ Hospital mortality } & \multirow[t]{2}{*}{ Cases } & \multirow{2}{*}{$\begin{array}{l}\text { Conversion } \\
\text { to } \\
\text { thoracotomy }\end{array}$} & \multicolumn{3}{|c|}{ Hospital mortality } & \\
\hline & & $\sim 30$ days & 31-90 days & $\begin{array}{l}\text { Total (including } \\
\text { after 91days } \\
\text { mortality) }\end{array}$ & & & $\sim 30$ days & 31-90days & $\begin{array}{l}\text { Total (including } \\
\text { after 91days } \\
\text { mortality) }\end{array}$ & \\
\hline $\begin{array}{l}\text { Salvage } \\
\text { surgery }\end{array}$ & 570 & $3(0.5)$ & $6(1.1)$ & $9(1.6)$ & 272 & $4(1.5)$ & $1(0.4)$ & $3(1.1)$ & $4(1.5)$ & 245 \\
\hline
\end{tabular}


performed in $89.1 \%$ (115/129), $81.5 \%$ (387/475), $51.6 \%$ (33/64), and $49.5 \%(102 / 206)$ of patients with esophagitis (including esophageal ulcer), hiatal hernia, benign tumors, and achalasia, respectively. On the other hand, 89.1\% (115/ 129) of patients with spontaneous rupture of the esophagus underwent open surgery. Hospital mortality rates after surgery for benign esophageal diseases had only been recorded for those with hiatal hernia and spontaneous rupture of the esophagus, with $8(1.7 \%)$ and $2(1.6 \%)$ patients succumbing to mortality within 30 days following surgery, respectively. Only $3(0.4 \%)$ among the 673 patients who underwent thoracoscopic and/or laparoscopic surgery died within 30 postoperative days, all of whom had hiatal hernia.

The most common tumor location for malignant esophageal diseases was the thoracic esophagus (Table 36). Among 6256 cases with esophageal malignancies, 2538 (40.6\%) and 3718 (59.4\%) underwent esophagectomy for superficial and advanced cancers, respectively. The 30-day and hospital mortality rates following esophagectomy were $0.4 \%$ and $0.6 \%$ for patients with superficial cancer and $1.0 \%$ and $1.8 \%$ for those with advanced cancer, respectively.

Among esophagectomy procedures, transthoracic esophagectomy via right thoracotomy or right thoracoscopy was most commonly adopted for patients with a superficial cancer $(1908 / 2538,75.2 \%)$ and advanced cancer (3045/ $3718,81.9 \%$ ) (Table 36). Transhiatal esophagectomy, which is commonly performed in Western countries, was adopted in only $15(0.6 \%)$ and $32(0.9 \%)$ patients with superficial and advanced cancer who underwent esophagectomy in Japan, respectively. Thoracoscopic and/or laparoscopic esophagectomy was utilized in $1832(72.2 \%)$ and 2311 (62.2\%) patients with superficial and advanced cancer, respectively. The number of patients who underwent thoracoscopic and/or laparoscopic surgery for superficial or advanced cancer has been increasing, whereas that of open surgery, especially for advanced cancer, has been decreasing annually (Fig. 4). Mediastinoscopic and robot-assisted esophagectomy and reconstruction were performed for 173 and 328 patients in 2018, respectively. The 30-day and hospital mortality rates following thoracoscopic and/or laparoscopic esophagectomy were $0.5 \%$ and $0.7 \%$ for patients with superficial cancer and $0.9 \%$ and $1.4 \%$ or those with advanced cancer, respectively (Table 36 ).

Detailed data collection regarding postoperative surgical and non-surgical complications have been initiated this year (Table 36). Overall, 1360 (21.7\%) of 6256 patients developed grade III or higher complications based on the Clavien-Dindo classification. Among surgical complications, anastomotic leakage and recurrent nerve palsy occurred in $13.0 \%$ and $13.2 \%$ of the patients and in approximately $20 \%$ and $24 \%$ of those who underwent mediastinoscopic esophagectomy, respectively. Among non-surgical postoperative complications, pneumonia occurred in $13.8 \%$ of the patients, $4.8 \%$ of whom underwent unplanned intubation. Mediastinoscopic esophagectomy seemed to be less likely to promote postoperative pneumonia compared to transthoracic (rt.) esophagectomy. Postoperative pulmonary embolism occurred in $0.7 \%$ of the patients.

Salvage surgery following definitive (chemo) radiotherapy was performed in 570 patients, with a 30 -day and hospital mortality rate of $0.5 \%$ and $1.6 \%$, respectively. Thoracoscopic and/or laparoscopic esophagectomy were performed in $272(47.7 \%)$ patients, both of which had comparable mortality rates (Table 37 ).

We aim to continue our efforts in collecting comprehensive survey data through more active collaboration with the Japan Esophageal Society and other related institutions.

Acknowledgements On behalf of the Japanese Association for Thoracic Surgery, the authors thank the Heads of the Affiliate and Satellite Institutes of Thoracic Surgery for their cooperation and the Councilors of the Japan Esophageal Society.

Open Access This article is licensed under a Creative Commons Attribution 4.0 International License, which permits use, sharing, adaptation, distribution and reproduction in any medium or format, as long as you give appropriate credit to the original author(s) and the source, provide a link to the Creative Commons licence, and indicate if changes were made. The images or other third party material in this article are included in the article's Creative Commons licence, unless indicated otherwise in a credit line to the material. If material is not included in the article's Creative Commons licence and your intended use is not permitted by statutory regulation or exceeds the permitted use, you will need to obtain permission directly from the copyright holder. To view a copy of this licence, visit http://creativecommons. org/licenses/by/4.0/.

\section{References}

1. Shimizu H, Okada M, Tangoku A, et al. Thoracic and cardiovascular surgery in Japan during 2017-Annual report by the Japanese Association for Thoracic Surgery. Gen Thorac Cardiovasc Surg. 2020;68:414-49.

2. Sakata R, Fujii Y, Kuwano H. Thoracic and cardiovascular surgery in Japan during 2008-Annual report by the Japanese Association for Thoracic Surgery. Gen Thorac Cardiovasc Surg. 2010;58:356-83.

3. Masuda M, Kuwano H, Okumura M, et al. Thoracic and cardiovascular surgery in Japan during 2013-Annual report by the Japanese Association for Thoracic Surgery. Gen Thorac Cardiovasc Surg. 2015;63:670-701.

4. Kazui T, Wada H, Fujita H. Thoracic and cardiovascular surgery in Japan during 2003-Annual report by the Japanese Association for Thoracic Surgery. Jpn J Thorac Cardiovasc Surg. 2005;53:517-36.

5. Kazui T, Osada H, Fujita H. Thoracic and cardiovascular surgery in Japan during 2004-Annual report by the Japanese Association for Thoracic Surgery. Jpn J Thorac Cardiovasc Surg. 2006;54:363-86.

Publisher's Note Springer Nature remains neutral with regard to jurisdictional claims in published maps and institutional affiliations. 


\section{Affiliations}

Committee for Scientific Affairs, The Japanese Association for Thoracic Surgery ${ }^{1} \cdot$ Hideyuki Shimizu $^{2}$. Morihito Okada ${ }^{3} \cdot$ Yasushi Toh $^{4} \cdot$ Yuichiro Doki $^{5}$ Shunsuke Endo ${ }^{6}$. Hirotsugu Fukuda ${ }^{7}$ Yasutaka Hirata ${ }^{8}$. Hisashi Iwata ${ }^{9}$. Junjiro Kobayashi ${ }^{10}$ - Hiraku Kumamaru ${ }^{11}$ - Hiroaki Miyata ${ }^{12}$ - Noboru Motomura ${ }^{13}$. Shoji Natsugoe ${ }^{14} \cdot$ Soji Ozawa $^{15} \cdot$ Yoshikatsu Saiki $^{16} \cdot$ Aya Saito $^{17} \cdot$ Hisashi Saji $^{18} \cdot$ Yukio Sato $^{19} \cdot$ Tsuyoshi Taketani $^{20} \cdot$ Kazuo Tanemoto $^{21}$ - Akira Tangoku ${ }^{22}$ - Wataru Tatsuishi ${ }^{23}$ - Hiroyuki Tsukihara ${ }^{24}$. Masayuki Watanabe ${ }^{25} \cdot$ Hiroyuki Yamamoto ${ }^{26} \cdot$ Kenji Minatoya $^{27} \cdot$ Kohei Yokoi $^{28} \cdot$ Yutaka Okita $^{29} \cdot$ Masanori Tsuchida $^{30} \cdot$ Yoshiki Sawa $^{31}$

1 Committee for Scientific Affairs, The Japanese Association for Thoracic Surgery, Tokyo, Japan

2 Department of Cardiovascular Surgery, Keio University, 35, Shinanomachi, Shinjuku-ku, Tokyo, Japan

3 Department of Surgical Oncology, Hiroshima University, Hiroshima, Japan

4 Department of Gastroenterological Surgery, National Hospital Organization Kyushu Cancer Center, Fukuoka, Japan

5 Department of Gastroenterological Surgery, Osaka University, Suita, Osaka, Japan

6 Department of Thoracic Surgery, Jichi Medical University, Shimotsuke, Japan

7 Department of Cardiac and Vascular Surgery, Dokkyo Medical University School of Medicine, Shimotsuga-gun, Tochigi, Japan

8 Department of Cardiac Surgery, The University of Tokyo Hospital, Tokyo, Japan

9 Department of General Thoracic Surgery, Gifu University Hospital, Gifu, Japan

10 Department of Cardiovascular Surgery, National Cerebral and Cardiovascular Center, Suita, Japan

11 Department of Healthcare Quality Assessment, Graduate School of Medicine, University of Tokyo, Tokyo, Japan

12 Department of Health Policy and Management, Keio University, Tokyo, Japan

13 Department of Cardiovascular Surgery, Toho University Sakura Medical Center, Sakura, Japan

14 Department of Digestive Surgery, Breast and Thyroid Surgery, Kagoshima University Graduate School of Medicine, Kagoshima, Japan

15 Department of Gastroenterological Surgery, Tokai University School of Medicine, Isehara, Japan

16 Division of Cardiovascular Surgery, Tohoku University Graduate School of Medicine, Sendai, Japan
17 Department of Cardiovascular Surgery, Toho University Sakura Medical Center, Sakura, Japan

18 Department of Chest Surgery, St. Marianna University School of Medicine, Kawasaki, Japan

19 Department of Thoracic Surgery, University of Tsukuba, Tsukuba, Japan

20 Department of Cardiovascular Surgery, Mitsui Memorial Hospital, Tokyo, Japan

21 Department of Cardiovascular Surgery, Kawasaki Medical School, Kurashiki, Japan

22 Department of Thoracic, Endocrine Surgery and Oncology, Institute of BioMedicine, Tokushima University Graduate School, Tokushima, Japan

23 Division of Cardiovascular Surgery, Department of General Surgical Science, Gunma University, Maebashi, Japan

24 Department of Cardiac Surgery, The University of Tokyo Hospital, Tokyo, Japan

25 Department of Gastroenterological Surgery, Cancer Institute Hospital of Japanese Foundation for Cancer Research, Tokyo, Japan

26 Department of Health Policy and Management, Keio University, Tokyo, Japan

27 Department of Cardiovascular Surgery, Graduate School of Medicine, Kyoto University, Kyoto, Japan

28 Chunichi Hospital, Nagoya, Japan

29 Cardio-Aortic Center, Takatsuki General Hospital, Takatsuki, Japan

30 Division of Thoracic and Cardiovascular Surgery, Niigata University Graduate School of Medical and Dental Sciences, Niigata, Japan

31 Department of Cardiovascular Surgery, Graduate School of Medicine, Osaka University, Osaka, Japan 